\title{
Theoretical study of the ground-state structures and properties of niobium hydrides under pressure
}

\author{
Guoying Gao, ${ }^{1,2}$ Roald Hoffmann,,${ }^{2, *}$ N. W. Ashcroft,,${ }^{3, *}$ Hanyu Liu, ${ }^{1}$ Aitor Bergara, ${ }^{4,5,6}$ and Yanming Ma ${ }^{1, *}$ \\ ${ }^{1}$ State Key Laboratory of Superhard Materials, Jilin University, Changchun 130012, People's Republic of China \\ ${ }^{2}$ Department of Chemistry and Chemical Biology, Cornell University, Ithaca, New York, 14853, USA \\ ${ }^{3}$ Laboratory of Atomic and Solid State Physics, Cornell University, Ithaca, New York, 14853, USA \\ ${ }^{4}$ Materia Kondentsatuaren Fisika Saila, Zientzia eta Teknologia Fakultatea, Euskal Herriko Unibertsitatea (UPV/EHU), 644 Postakutxatila, \\ 48080 Bilbo, Basque Country, Spain \\ ${ }^{5}$ Donostia International Physics Center (DIPC), Paseo de Manuel Lardizabal, 20018, Donostia, Basque Country, Spain \\ ${ }^{6}$ Centro de Fisica de Materiales CSIC-UPV/EHU, 1072 Posta kutxatila, E-20080 Donostia, Basque Country, Spain
}

(Received 24 February 2013; revised manuscript received 4 October 2013; published 12 November 2013)

\begin{abstract}
As part of a search for enhanced superconductivity, we explore theoretically the ground-state structures and properties of some hydrides of niobium over a range of pressures and particularly those with significant hydrogen content. A primary motivation originates with the observation that under normal conditions niobium is the element with the highest superconducting transition temperature $\left(T_{c}\right)$, and moreover some of its compounds are metals again with very high $T_{c}$ 's. Accordingly, combinations of niobium with hydrogen, with its high dynamic energy scale, are also of considerable interest. This is reinforced further by the suggestion that close to its insulator-metal transition, hydrogen may be induced to enter the metallic state somewhat prematurely by the addition of a relatively small concentration of a suitable transition metal. Here, the methods used correctly reproduce some ground-state structures of niobium hydrides at even higher concentrations of niobium. Interestingly, the particular stoichiometries represented by $\mathrm{NbH}_{4}$ and $\mathrm{NbH}_{6}$ are stabilized at fairly low pressures when proton zero-point energies are included. While no paired $\mathrm{H}_{2}$ units are found in any of the hydrides we have studied up to $400 \mathrm{GPa}$, we do find complex and interesting networks of hydrogens around the niobiums in high-pressure $\mathrm{NbH}_{6}$. The $\mathrm{Nb}-\mathrm{Nb}$ separations in $\mathrm{NbH}_{n}$ are consistently larger than those found in $\mathrm{Nb}$ metal at the respective pressures. The structures found in the ground states of the high hydrides, many of them metallic, suggest that the coordination number of hydrogens around each niobium atom grows approximately as $4 n$ in $\mathrm{NbH}_{n}(n=1-4)$, and is as high as 20 in $\mathrm{NbH}_{6} . \mathrm{NbH}_{4}$ is found to be a plausible candidate to become a superconductor at high pressure, with an estimated $T_{c} \sim 38 \mathrm{~K}$ at $300 \mathrm{GPa}$.
\end{abstract}

DOI: 10.1103/PhysRevB.88.184104

PACS number(s): 74.62.Fj, 74.10.+v, 74.25.Jb

\section{INTRODUCTION}

Transition metal hydrides have had a rich history in the materials sciences for the last 50 years or more. Aside from the intrinsic interest in the metal-hydrogen bond and the electron density around hydrogen in solid-state hydrides, there is the known capacity of some metals (e.g., Y, Rh, Pd, Pt) and alloys (e.g., $\mathrm{LaNi}_{5}, \mathrm{Mg}_{2} \mathrm{Ni}, \mathrm{FeTi}$ ) to absorb relatively large amounts of hydrogen. This property has led to the real and potential use of metal hydrides as hydrogen storage materials. Hydrogen, in metals in hydridic interstitial form, modifies significantly the electronic properties of both the separated elements; so PdH at $P=1 \mathrm{~atm}$ is a superconductor with a transition temperature $T_{c}$ of about $9 \mathrm{~K}$ (and an unusual isotope effect), ${ }^{1}$ while $\mathrm{H}_{2}$ is an insulator at $1 \mathrm{~atm}$, and Pd is a metal but not a superconductor.

Also the quest for metallic hydrogen under pressure has also involved several theoretical groups in the examination of metal hydrides; particularly, we mention here investigations of alkali and alkaline earth hydrides, ${ }^{2-5}$ group 14 hydrides, ${ }^{6-8}$ and the tungsten hydrides, ${ }^{9}$ some of these carried out by our own research groups. We were led to look at the niobium hydrides in particular by the following additional observation: it is well known that the transition metal $\mathrm{Nb}$ holds the record for the highest superconducting transition temperature $T_{c}(9.3 \mathrm{~K})$ of an element at normal pressure,$^{10}$ and compounds of $\mathrm{Nb}$, such as $\mathrm{Nb}_{3} \mathrm{Ge}(23 \mathrm{~K}),{ }^{11} \mathrm{NbC}(11 \mathrm{~K}),{ }^{12}$ and $\mathrm{NbN}(16 \mathrm{~K}),{ }^{13}$ also have the highest $T_{c}$ values for their respective classes. There is nothing obvious that distinguishes $\mathrm{Nb}$ in the key factors that enter, say, into a BCS estimate of the $T_{c}$ for $\mathrm{Nb}, \mathrm{NbN}, \mathrm{NbC}$, $\mathrm{NbO}$, or $\mathrm{Nb}_{3} \mathrm{Ge}$. Yet, prompted in equal measure by what may be an as yet not understood coincidence and also by an impression (occasioned by previous studies in our groups) that the enthalpies or chemical bonding in transition metal hydrides is inherently interesting, and finally, a desire to extend our knowledge of chemically and physically diverse matter under pressure, we undertook an extensive exploration of niobium hydrides, particularly those of lower to intermediate hydrogen content. A further motivating factor of some importance for the present investigations is the suggestion ${ }^{14}$ that if upon steady densification hydrogen is close to becoming a ground-state metal, then the addition of a small concentration of a second element, especially a transition metal, could substantially reduce the overall metallization pressure. The result would then be a very hydrogen rich binary metal with the possibility of a high superconducting transition temperature reflecting the dominance of hydrogen itself.

Throughout the paper, we will be making constant reference to "hydrides" and especially to the movement and placement of hydrogen "atoms." In large part, this latter terminology is simply following common usage in the literature though it should be made clear that in the condensed and high pressure environment in which we are looking what is being moved is, of course a proton. However, this is immediately accompanied by an electron response density, and an associated motion of electrons, as arises within the Born-Oppenheimer separation of timescales. 
Several hydrides of niobium in molecular form are known and their properties are an instructive guide to what we may expect in truly extended systems. These metastable molecules have been formed in near isolation and characterized in a neon matrix by Wang and Andrews; ${ }^{15}$ they obtained the molecular complexes $\mathrm{NbH}_{2}, \mathrm{NbH}_{4}, \mathrm{NbH}_{4}\left(\mathrm{H}_{2}\right), \mathrm{NbH}_{4}\left(\mathrm{H}_{2}\right)_{2}, \mathrm{NbH}_{4}\left(\mathrm{H}_{2}\right)_{3}$, and even $\mathrm{NbH}_{4}\left(\mathrm{H}_{2}\right)_{4}$ by reacting laser ablated niobium with molecular hydrogen in excess neon, and then characterized them spectroscopically. ${ }^{15}$ While stable against atomization, all these molecular niobium hydrides have large positive heats of formation relative to the separated elements and small barriers to oligomerization; thus they do not survive any warming up of the matrix in which they are being observed.

Again, as a preface to what follows, it is also useful to turn to even more complex arrangements, there are a number of kinetically persistent organometallic hydrides of niobium, ${ }^{16}$ which are of some comparative interest. In Fig. S1 in Supplementary Material, ${ }^{17}$ we show a selection of these ${ }^{18-20}$ and we note that in these systems, hydrogen is terminally bonded to niobium, and also bridging two niobiums. X-ray determined crystal structures do not locate hydrogens reliably, but neutron diffraction experiments (less plentiful, of course) do so; we then see $\mathrm{Nb}-\mathrm{H}$ separations of $\sim 1.80 \AA$ for terminal $\mathrm{Nb}-\mathrm{H}$, and 1.9-2.0 $\AA$ for bridging hydrogens. The relevance of these to the extended niobium hydrides we study in this paper is that the $\mathrm{Nb}-\mathrm{H}$ separations in these molecules are a guidepost to what we might expect to find in the extended (dense) hydrides at low pressures.

Moving on now to these possibly extended structures, solid $\mathrm{Nb}$ reacts directly with hydrogen at atmospheric pressure and at room temperature to form usually nonstoichiometric hydrides, which at one atmosphere appear to have a limiting composition of $\mathrm{NbH}_{x}(x \leqslant 0.9)$. Experimental studies of the structure of these materials find that the hydrogen ions are located in the (approximately) tetrahedral $(T)$ interstitial sites in a bcc $\mathrm{Nb}$ sublattice (see Fig. S2). ${ }^{17}$ A number of forms of $\mathrm{NbH}_{x}(x<1)$ have been proposed. Above room temperature (at about $350 \mathrm{~K}$ ), disordered $\alpha$ and $\alpha^{\prime}$ phases of $\mathrm{NbH}_{x}(x \leqslant 1)$ are stable, with the hydrogen ions then distributed randomly on the $T$ sites. The $\alpha$ phase is also stable at very low hydrogen concentrations and lower temperatures.

At and below room temperature and at one atmosphere, the hydrogen atoms order in the $\beta$-phase structure, or into a mixture of $\beta$ and $\gamma$ phases. $^{21}$ The $\beta$ phase is deduced to be face- centered orthorhombic from both neutron ${ }^{22}$ and also electron diffraction ${ }^{23}$ experiments; the structure for the $\gamma$ phase (approximate composition of $\mathrm{NbH}_{0.9}$ ) was proposed by $\mathrm{Hauck}^{24}$ to be pseudocubic, but there is no experimental confirmation of this to date.

At about 2 atm, $\mathrm{NbH}_{2}$ (the $\delta$ phase) has been synthesized by a direct reaction ${ }^{25}$ of hydrogen with $\mathrm{Nb}$ metal, and also by using an aqueous electrolytic method. ${ }^{26} \mathrm{NbH}_{2}$ has the common $\mathrm{CaF}_{2}$ type structure, where the $\mathrm{Nb}$ metal forms an fcc structure and the $\mathrm{H}$ atoms occupy all the $T$ sites of the fcc lattice. Higher hydrides of niobium have apparently not been synthesized at one atmosphere or so far at high pressure. Experimentally, $\mathrm{NbH}_{2}$ is certainly a metal but has not been observed to exhibit superconductivity above $1.2 \mathrm{~K}$ at one atmosphere. ${ }^{27}$

Given this experimental overview, our theoretical exploration of the $\mathrm{Nb}-\mathrm{H}$ phase diagram, and particularly the crucial underlying electronic aspects begins with stoichiometric $\mathrm{NbH}$, as well as $\mathrm{Nb}_{4} \mathrm{H}_{3}$, an approximation to the nonstoichiometric $\mathrm{NbH}_{x}(x<1)$ phase experimentally known. ${ }^{28}$ The study then continues to the also available (at 1 atm) $\mathrm{NbH}_{2}$. From these we proceed to the as yet hypothetical $\mathrm{NbH}_{3}, \mathrm{NbH}_{4}, \mathrm{NbH}_{5}$, and $\mathrm{NbH}_{6}$ stoichiometries, all studied over a range of pressures up to $500 \mathrm{GPa}$, with relative compressions reaching $V / V_{0} \sim 2.2$. (We note here that in a succeeding paper, even higher hydrides are treated.) The calculational methodology we have used is fully described in the computational details. In particular, we examine in detail the optimum static structures as a function of pressure, as well as their dynamical stability, and the corresponding electronic band structures and superconductivity of the stable phases predicted. We find that all the niobium hydrides studied are metallic and $\mathrm{NbH}_{4}$ is predicted to be a potential candidate for plausible superconductor.

\section{COMPUTATIONAL DETAILS}

We extensively searched for $\mathrm{NbH}_{n} \quad(n=0.75$, and 1-6) ground-state structures using Crystal structure AnaLYsis by Particle Swarm Optimization methodology, ${ }^{29,30}$ as implemented in the CALYPSO code. ${ }^{31}$ This method has been benchmarked on a variety of known systems and has made several successful predictions of high pressure structures of, for example, $\mathrm{Li}, \mathrm{Mg}$, and $\mathrm{Bi}_{2} \mathrm{Te}_{3} .{ }^{32-34}$ Our structure searches with system sizes containing up to 8 formula units (f.u.) per simulation cell were performed at pressures of 15-400 GPa. Each generation contains 30-40 structures (the larger the system the larger the number of structures). The first generation is produced randomly, and then these structures are optimized. For the next generation, $60 \%$ of the structures are generated from the best structures in the previous generation by particle swarm optimization and the others by random choice. These are then reoptimized and the previous steps repeated until convergence. We usually perform 30-50 generations (depending on the size of the system) to achieve a converged structure.

The underlying ab initio structural relaxations were carried out using density functional theory using the Perdew-BurkeErnzerhof exchange-correlation functional ${ }^{35}$ as implemented in the VASP code. ${ }^{36}$ The frozen-core all-electron projectoraugmented wave method ${ }^{37}$ was adopted and for hydrogen the $1 s^{\prime}$ cut-off radius was $1.1 a_{0}$ (reduction to $0.8 a_{0}$ does not change our conclusions). For niobium the radius for $4 p^{6} 4 d^{4} 5 s^{1}$ was taken as $2.75 a_{0}$. An energy cutoff of $600 \mathrm{eV}$ and appropriate Monkhorst-Pack ${ }^{38} \boldsymbol{k}$ meshes were chosen to ensure that enthalpy calculations were well converged to better than $1 \mathrm{meV} /$ formula unit (f.u.). The phonon calculations were carried out by using a supercell approach ${ }^{39}$ within the PHONOPY code. ${ }^{40}$ We used $2 \times 3 \times 2,2 \times 2 \times 2,3 \times 3 \times 3$, and $2 \times 2 \times 2$ supercells for Pnma- $\mathrm{NbH}_{2}, I-43 d-\mathrm{NbH}_{3}$, I $4 / \mathrm{mmm}^{-\mathrm{NbH}_{4}}$, and $\mathrm{Cmmm}-\mathrm{NbH}_{6}$, respectively.

Electron-phonon coupling (EPC) calculations utilized the plane-wave pseudopotential method within density functional perturbation theory as within the Quantum-ESPRESSO source code. ${ }^{41}$ A $6 \times 6 \times 4$ for $C c c m-N b H, 8 \times 8 \times 8$ for $F m$ $3 m-\mathrm{NbH}_{2}$, and $6 \times 6 \times 6$ for $I 4 / m m m-\mathrm{NbH}_{4} \boldsymbol{q}$-point mesh in the first Brillouin zone (BZ) was used in the EPC calculation. A MP grid of $36 \times 36 \times 24,48 \times 48 \times 48$, and $36 \times 36$ 
$\times 36$ was used to ensure $\boldsymbol{k}$-point sampling convergence with Gaussians of width $0.03 \mathrm{Ry}$ (408 meV) for $\mathrm{NbH}, \mathrm{NbH}_{2}$, and $\mathrm{NbH}_{4}$, respectively, which approximates the zero-width limits in calculations of the EPC parameter $\lambda$. Here, $\lambda$ is the first reciprocal moment of the spectral function $\alpha^{2} F(\omega)$,

$$
\lambda=2 \int_{0}^{\infty} \frac{\alpha^{2} F(\omega)}{\omega} d \omega \approx \sum_{\boldsymbol{q} j} \lambda_{\boldsymbol{q} j} w(\boldsymbol{q}),
$$

where $w(\boldsymbol{q})$ is the weight (the weights account for the symmetries of the BZ) of a $\boldsymbol{q}$ point in the first BZ, and the EPC spectral function $\alpha^{2} F(\omega)$ is expressed in terms of the phonon linewidth $\gamma_{\boldsymbol{q} j}$ arising from electron-phonon coupling: ${ }^{42-44}$

$$
\alpha^{2} F(\omega)=\frac{1}{2 \pi \mathrm{N}_{f}} \sum_{\boldsymbol{q} j} \frac{\gamma_{\boldsymbol{q} j}}{\omega_{\boldsymbol{q} j}} \delta\left(\omega-\omega_{\boldsymbol{q} j}\right) w(\boldsymbol{q}),
$$

In equation (2), $N_{f}$ is the electronic density of electron states at the Fermi level. The linewidth $\gamma_{q j}$ of a phonon mode $j$ at wave vector $\boldsymbol{q}$, arising from EPC is given by

$$
\begin{aligned}
\gamma_{\boldsymbol{q} j}= & 2 \pi \omega_{\boldsymbol{q} j} \sum_{n m} \int \frac{d^{3} \mathbf{k}}{\Omega_{\mathbf{B Z}}}\left|\mathbf{g}_{\boldsymbol{k} n, \boldsymbol{k}+\boldsymbol{q} m}^{j}\right|^{2} \\
& \times \delta\left(\varepsilon_{\boldsymbol{k} n}-\varepsilon_{\boldsymbol{F}}\right) \delta\left(\varepsilon_{\boldsymbol{k}+\boldsymbol{q} m}-\varepsilon_{\boldsymbol{F}}\right),
\end{aligned}
$$

where the sum is over the first BZ, with $\Omega_{\mathrm{BZ}}$ as the volume of the BZ, and $\varepsilon_{\boldsymbol{k} n}$ are the energies of bands (measured with respect to the Fermi level $\varepsilon_{\boldsymbol{F}}$ ) at point $\boldsymbol{k}$. Here, $\mathbf{g}_{\boldsymbol{k} n, \boldsymbol{k}+\boldsymbol{q} m}^{j}$ is the electron-phonon matrix element for scattering from an electron in band $n$ at wave vector $\boldsymbol{k}$ state to band $m$ at wave vector $\boldsymbol{k}+\boldsymbol{q}$ via a phonon with wave vector $\boldsymbol{q}$, and can be expressed as $\mathbf{g}_{\boldsymbol{k} n, \boldsymbol{k}+\boldsymbol{q} m}^{j}=\left(\frac{\hbar}{2 M \omega_{q j}}\right)^{1 / 2}\left\langle\varphi_{n, \boldsymbol{k}}\left|\delta V_{\boldsymbol{q}} \boldsymbol{e}_{\boldsymbol{q} j}\right| \varphi_{m, \boldsymbol{k}+\boldsymbol{q}}\right\rangle$, where $M$ is the mass of the ion, $\delta V_{\boldsymbol{q}}$ is the first derivative of the effective external potential with respect to the ions' displacements, and $\boldsymbol{e}_{\boldsymbol{q} j}$ is the eigenvector of phonon branch $j$ at wave vector $\boldsymbol{q}$.

The superconducting transition temperature $T_{c}$ has been estimated with the use of the Allen-Dynes modified McMillan equation: ${ }^{45}$

$$
k_{B} T_{C}=\frac{\hbar \omega_{\ln }}{1.2} \exp \left[-\frac{1.04(1+\lambda)}{\lambda-\mu^{*}(1+0.62 \lambda)}\right],
$$

where $\lambda$ is defined above, $\omega_{\ln }$ is the logarithmic average of the phonon frequencies

$$
\omega_{\ln }=\omega_{0} \exp \left[\frac{2}{\lambda} \int \frac{\alpha^{2} \mathrm{~F}(\omega)}{\omega} \ln \frac{\omega}{\omega_{0}} d \omega\right]
$$

where $d \omega_{\ln } / d \omega_{0}=0$, indicating a basic formal independence on the value chosen for $\omega_{0}$. However, following common literature usage we take $\omega_{0}=1 \mathrm{THz}$. Note that $\omega_{\mathrm{ln}}, \omega_{0}$ and $\omega$ are also often used as ordinary frequencies not angular frequencies and $\mu^{*}$, the effective retarded Coulomb repulsion parameter, is defined as $\frac{1}{\mu^{*}}=\frac{1}{\mu}+\ln \left(\frac{\omega_{\mathrm{el}}}{\omega_{\mathrm{ph}}}\right)$, where $\mu$ is the direct Coulomb repulsion between paired electrons, $\omega_{\mathrm{el}}$ corresponds to the electron plasma frequency, and $\omega_{\mathrm{ph}}$ is the high-frequency cutoff in the EPC spectral function $\alpha^{2} F(\omega)^{.45}$ It remains challenging to directly derive an accurate $\mu^{*}$ from first-principles theory but an upper bound on $\mu^{*}$ is estimated to be $0.25 .^{42}$ For hydrides, $\mu^{*}=0.1-0.13$ has been widely used in $T_{c}$ estimates. In the following, we used $\mu^{*}=0.1$ and 0.13 in the present $T_{c}$ estimates.

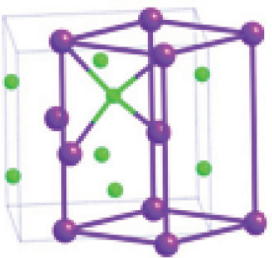

(a) $\mathrm{Cccm}$

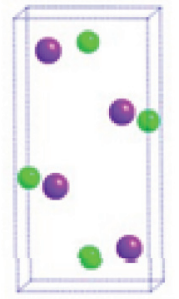

(d) Pnma

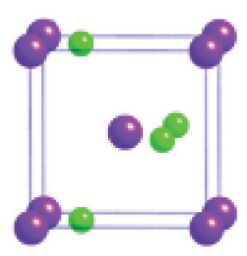

(b) I-4m2

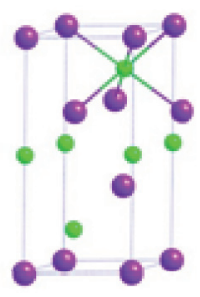

(e) $\mathbf{R}-\mathbf{3 m}$

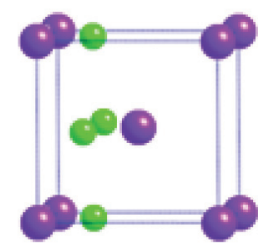

(c) $\mathrm{P} 4 / \mathrm{nmm}$

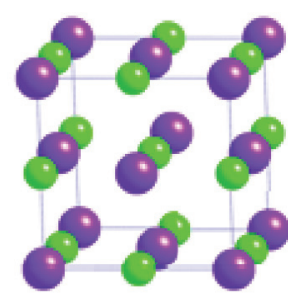

(f) $\mathbf{F m}-\mathbf{3 m}$
FIG. 1. (Color online) Various predicted ground-state static highpressure structures for $\mathrm{NbH}$. Here (and throughout), Nb's are depicted as large purple spheres and H's as smaller green ones.

\section{RESULTS AND DISCUSSION}

\section{A. $\mathbf{N b H}$}

The structural search at $15 \mathrm{GPa}$ for the stoichiometric $\mathrm{NbH}$ predicts a static orthorhombic $\operatorname{Cccm}(Z=4)$ arrangement [see Fig. 1(a)], where the $\mathrm{Nb}$ atoms can be seen to form a distorted bcc structure with the hydrogen atoms occupying two of the $T$ interstices of a bcc Nb lattice. We also obtained metastable $I-4 m 2[Z=2$, Fig. 1(b)], P4/nmm [ $Z=2$, Fig. 1(c)], and Pnma $[Z=4$, Fig. 1(d)] structures. Most of these structures realize different occupancies of the $T$ sites of a bcc lattice and are very close to each other in enthalpy at $1 \mathrm{~atm}$, in fact within $0.03 \mathrm{eV}$ per formula unit. This value is actually smaller than the zero-point energies (ZPE) as calculated in the harmonic approximation for the individual structures, which are of the order of $0.129 \mathrm{eV}$, and are very similar for all of them (this important matter is taken up below). It is also quite close to typical thermal energies.

Our predicted metastable $I-4 m 2$ structure for $\mathrm{NbH}$ happens to be identical to that found for an earlier theoretically proposed $\gamma$ phase. $^{21}$ We made a preliminary estimate of the enthalpy barrier for the displacement of hydrogens that converts one structure to the other ( $I-4 m 2$ and $P 4 / n m m)$. This barrier is calculated to be low $(\sim 0.65 \mathrm{eV})$ at 1 atm; these $\mathrm{NbH}$ structures are therefore likely to interconvert easily at low pressures. ${ }^{46}$

Above $100 \mathrm{GPa}$, a rhombohedral centered hexagonal $R-3 m$ $(Z=3)$ structure [see Fig. 1(e)] is predicted for $\mathrm{NbH}$, which is then followed, but at much higher pressures, by a preferred face-centered cubic $F m-3 m$ ( $\mathrm{NaCl}$ type, $Z=4)$ structure [see Fig. 1(f)]. In this geometry, all the hydrogen atoms occupy the octahedral $(O)$ interstices, not tetrahedral (see Fig. S3 for the familiar site environment notation). ${ }^{17}$ As pressure increases the evolution of interstitial site occupation in $\mathrm{NbH}$, from $T$ to $O$, is again consistent with previous work, ${ }^{47}$ and also the anticipated generalization that the number of neighbors of a hydrogen-occupied site that is likely to increase with increasing pressure. 


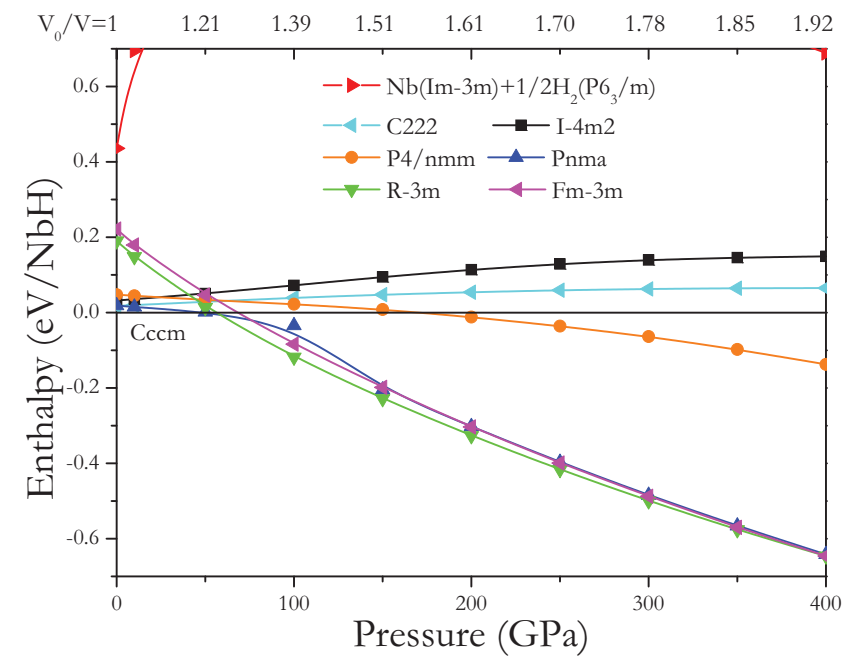

FIG. 2. (Color online) Ground-state enthalpy curves per formula unit as a function of pressure for $\mathrm{NbH}$ (static calculations), with respect to that for the $\mathrm{Cccm}$ structure. The decomposition enthalpies for $\mathrm{NbH}$ to $\mathrm{Nb}+\mathrm{H}_{2}$ are also presented. We considered the Im$3 m$ structure for separated pure solid $\mathrm{Nb}^{47}$ and $P 6_{3} / m$ for $\mathrm{H}_{2}{ }^{48}$ The relative compression $\left(V_{0} / V\right)$ is also given for the corresponding pressures (upper horizontal axis).

Figure 2 shows the calculated ground-state static enthalpy per formula unit for the various structures at different pressures. Note especially the five structures enthalpically close to each other at $P=1 \mathrm{~atm}$; they correspond to the various occupations of $T$ sites in the bcc structure, as we discussed earlier. At around $60 \mathrm{GPa}\left(V_{0} / V=1.27\right)$ these are replaced by $R-3 m$ and $F m-3 m$ structures, which remain enthalpically close to each other over a large pressure range. Our predicted ground-state structures for $\mathrm{NbH}$ are all enthalpically stable with respect to separation into the elements themselves over the whole pressure range studied.

\section{B. $\mathrm{Nb}_{4} \mathrm{H}_{3}$}

As discussed above, at 1 atm the experimentally synthesized niobium hydrides are usually observed to be nonstoichiometric, with the ratio of the concentration $\mathrm{H} / \mathrm{Nb}$ being less than 1 . To explore the consequences of a possible departure from stoichiometry, we now take $\mathrm{NbH}_{0.75}\left(\mathrm{Nb}_{4} \mathrm{H}_{3}\right)$ as an example, which is also synthesized by experiments. ${ }^{49,50}$

At $1 \mathrm{~atm}$, we find that the primitive static monoclinic $P 2$ structure has the lowest enthalpy $[Z=1$, Fig. 3(a)]. This structure can also be described as a slightly distorted bcc $\mathrm{Nb}$ lattice but now with hydrogen atoms partially occupying certain $T$ interstitial sites [see the representation at the right in Fig. 3(a)]. At 100 and $400 \mathrm{GPa}$, we predict a stabilization (these are all ground state calculations) of a base-centered monoclinic $C 2 / m$ structure $[Z=2$, Fig. 3(b)], in which all the hydrogen atoms change from $T$ to $O$ interstices.

Figure 4 shows that static $\mathrm{Nb}_{4} \mathrm{H}_{3}$ below $50 \mathrm{GPa}$ is stable in the $P 2$ structure with respect to decomposition into $\mathrm{Nb}+\mathrm{H}_{2}$ and $\mathrm{NbH}+\mathrm{Nb}$. This finding is in satisfactory agreement with the observed off- $\mathrm{NbH}$ stoichiometry of phases synthesized at $P=1$ atm. $\mathrm{Nb}_{4} \mathrm{H}_{3}$ becomes unstable to decomposition or disproportionation into $\mathrm{NbH}+\mathrm{Nb}$ above $50 \mathrm{GPa}$, but still

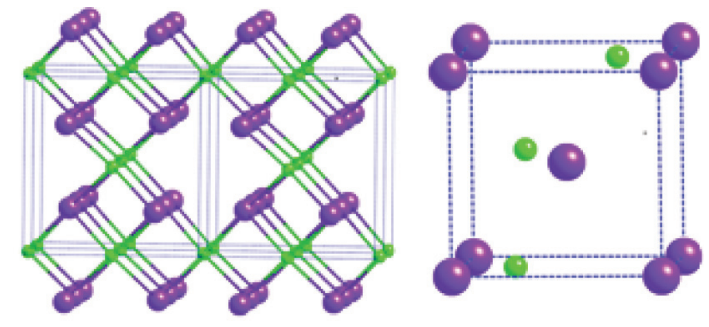

(a) P2

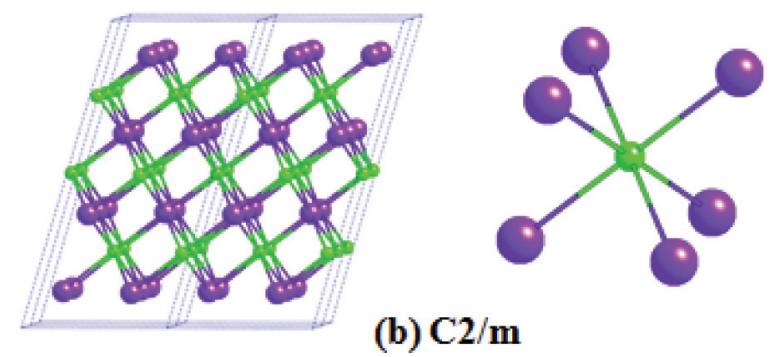

FIG. 3. (Color online) Predicted ground-state and static highpressure structures for $\mathrm{Nb}_{4} \mathrm{H}_{3}$ (a) $P 2$ at 1 atm and (b) at $400 \mathrm{GPa}$.

remains stable relative to the separated elements niobium and hydrogen.

\section{C. $\mathrm{NbH}_{2}$}

For static ground-state $\mathrm{NbH}_{2}$, at 1atm, 15 and $50 \mathrm{GPa}$, we find that the fluorite $F m-3 m$ structure $[Z=4$, Fig. 5 (a) $]$ is the most stable enthalpically, which is in good agreement with the experimental results these again being at room temperature. ${ }^{25}$ Above $50 \mathrm{GPa}$, an orthorhombic Pnma structure $[Z=4$, Fig. 5(b)] is then preferred. This structure is also the highpressure phase of $\mathrm{WH}_{2}{ }^{9}$ and certain alkali-earth dihydrides such as $\mathrm{CaH}_{2}{ }^{51,52}$ and $\mathrm{BaH}_{2} \cdot{ }^{53}$ We also find several metastable

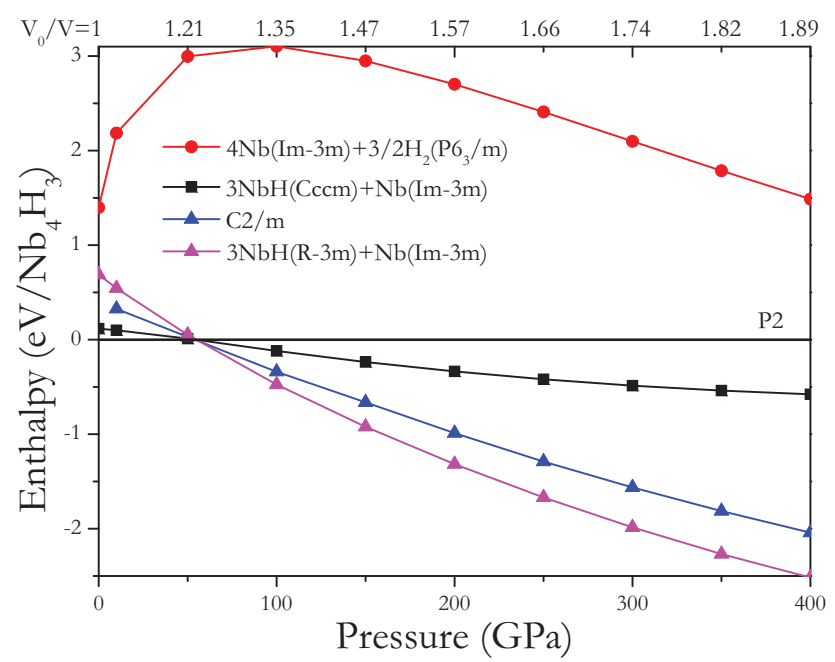

FIG. 4. (Color online) Ground-state static enthalpy curves per formula unit as a function of pressure with respect to the predicted $P 2$ structure for static $\mathrm{Nb}_{4} \mathrm{H}_{3}$. The decomposition enthalpies for $\mathrm{Nb}_{4} \mathrm{H}_{3}$ with respect to $\mathrm{Nb}+\mathrm{H}_{2}$ and $\mathrm{NbH}+\mathrm{Nb}$ are also presented. We have again considered the $\mathrm{Im}-3 \mathrm{~m}$ structure for pure solid $\mathrm{Nb}, \mathrm{P}_{3} / \mathrm{m}$ for $\mathrm{H}_{2}$ and the predicted $C c c m$ and $R-3 m$ structures for $\mathrm{NbH}$. The relative compression $\left(V_{0} / V\right)$ is indicated at the top of the figure. 


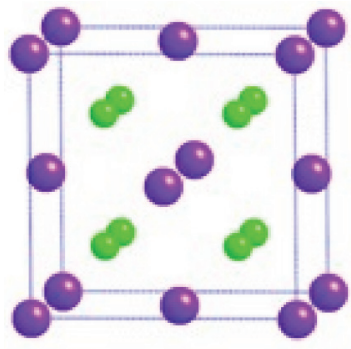

(a) Fm-3m

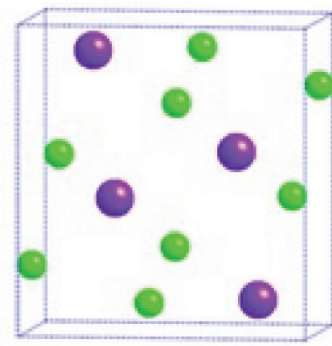

(b) Pnma
FIG. 5. (Color online) Predicted ground-state static structures for $\mathrm{NbH}_{2}$.

$P-3 m 1, P 4 / n m m$, and Pmn 21 structures. ${ }^{17}$ In the low-pressure $F m-3 m$ structure, all $\mathrm{H}$ atoms occupy the $T$ sites, while in the high-pressure Pnma phase, they are located at both $T$ and $O$ sites.

Figure 6 shows the evolution of the ground-state enthalpies with pressure. Our results indicate that static $\mathrm{NbH}_{2}$ is enthalpically stable with respect to decomposition into $\mathrm{Nb}+\mathrm{H}_{2}$ and $\mathrm{NbH}+\mathrm{H}_{2}$, respectively, at one atmosphere and also under pressure.

The consistency of the geometries we find for $\mathrm{NbH}, \mathrm{Nb}_{4} \mathrm{H}_{3}$, and $\mathrm{NbH}_{2}$ with room temperature experiment at $P=1$ atm now encourages us to examine other stoichiometries. As we will see, these may indeed be stable but in fact only at elevated pressures.

\section{D. $\mathrm{NbH}_{3}$}

This composition is not known experimentally at one atmosphere nor, as yet, under pressure. However, there are many known $\mathrm{AH}_{3}$-type compounds, for example, $\mathrm{YH}_{3}$ and $\mathrm{AlH}_{3}$, as well as the classically bonded dimers of $\mathrm{SiH}_{3}$

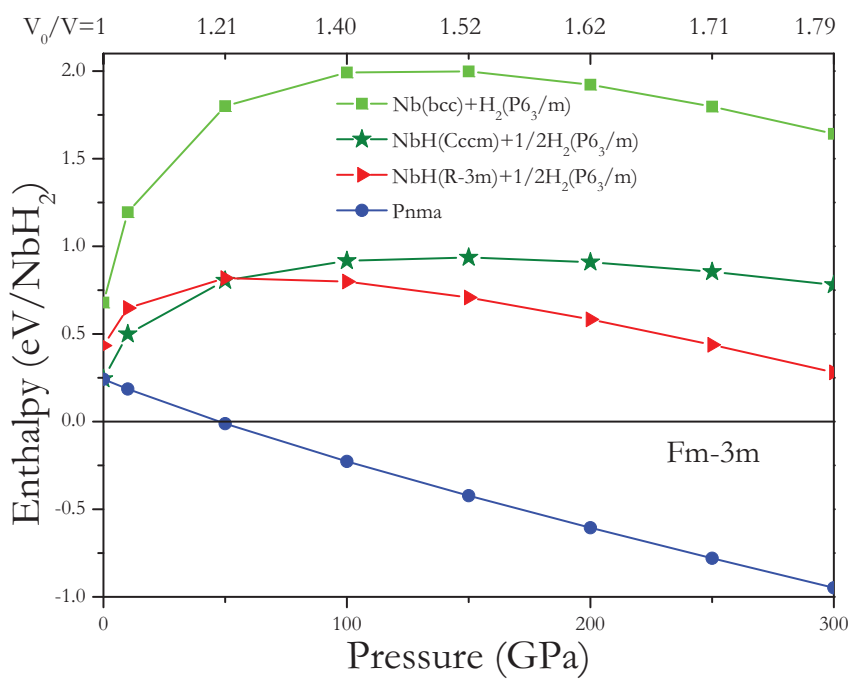

FIG. 6. (Color online) Static ground-state static enthalpy curves per formula unit as a function of pressure with respect to the predicted Fm-3m structure for static $\mathrm{NbH}_{2}$. The decomposition enthalpies for $\mathrm{NbH}_{2}$ with respect to $\mathrm{Nb}+\mathrm{H}_{2}$ and $\mathrm{NbH}+\mathrm{H}_{2}$ are also presented. We have again considered the $\mathrm{Im}-3 \mathrm{~m}$ structure for pure solid $\mathrm{Nb}$ and $\mathrm{P6}_{3} / m$ for $\mathrm{H}_{2}$. The relative compression $\left(V_{0} / V\right)$ is again indicated. (a) $\mathbf{F m}-3 \mathrm{~m}$

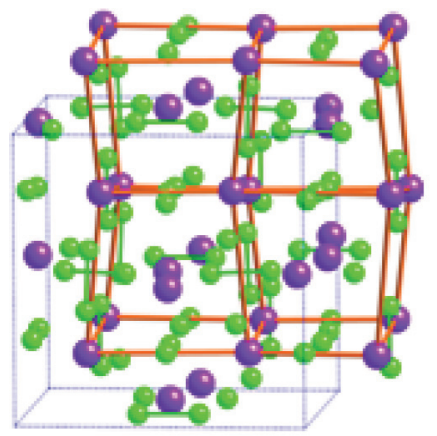

(c) I-43d

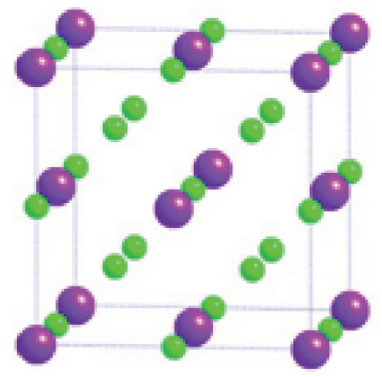

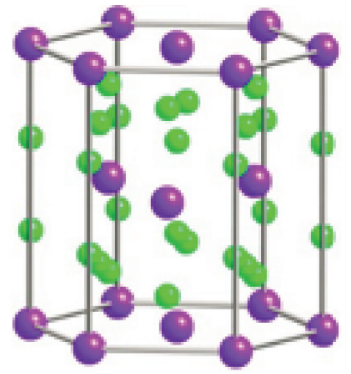

(b) $\mathrm{P} 63 / \mathrm{mmc}$

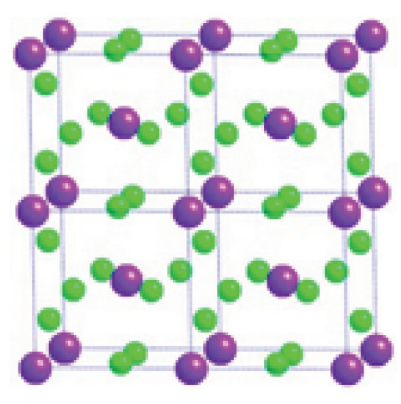

(d) Pm-3n $(2 \times 2 \times 1)$
FIG. 7. (Color online) Predicted static ground-state high-pressure $\mathrm{Fm}-3 \mathrm{~m}, \mathrm{P}_{3} / \mathrm{mmc}$, and $\mathrm{I}-43 \mathrm{~d}$ ground-state structures for $\mathrm{NbH}_{3}$.

and $\mathrm{CH}_{3}$. The observed structures are $\mathrm{Fm}-3 m$ for $\mathrm{YH}_{3},{ }^{54}$ $P m-3 n$ for $\mathrm{AlH}_{3},{ }^{55}$ and the predicted low- and high- pressure structures $\mathrm{Cmcm}, \mathrm{PG}_{3} / m m c$, and $C 2 / m \mathrm{YH}_{3},{ }^{54}$ and $P m-3 m$ for $\mathrm{Si}_{2} \mathrm{H}_{6} \cdot{ }^{56}$ Among these structures, the $\mathrm{Fm}-3 \mathrm{~m}$ and $P 6_{3} / m m c$ structures can actually be obtained by placing $\mathrm{H}$ atoms in all the $T$ and $O$ sites of an fcc and hcp niobium lattices, respectively [see Figs. 7(a) and 7(b)]. Through the use of crystal structure prediction algorithms, we are then also led to these structures, as well as to a new $I-43 d$ structure.

The stability relationships are pretty clear from Fig. 8, which summarizes the enthalpy of various structures of static $\mathrm{NbH}_{3}$. The reference line is the simple fully occupied fcc lattice, $F m-3 m$, mentioned above. At $P=1$ atm $\mathrm{NbH}_{3}$ is not stable relative to decomposition into the dihydride. But at $P>33 \mathrm{GPa}$ a stable ground-state $\mathrm{NbH}_{3}$ structure does in fact emerge, a body-centered cubic $I-43 d(Z=16$, Pearson symbol cI64) one [see Fig. 7(c)]. This is the well-known $\mathrm{Cu}_{3}$ As arrangement and can also be seen as a distorted $P m-3 n$ $\left(\mathrm{Nb}_{3} \mathrm{Sn}\right.$-type) structure [see Fig. 7(d)]. It is interesting to note that the locations of niobiums in the metal sublattice are similar to those in the high pressure phase $(c I 16)$ of $\mathrm{Li}$, and that the $\mathrm{H}$ atoms occupy two of the $T$ interstitial positions in each plane of the slightly distorted bcc-Nb metal lattice.

The shortest separation of two $\mathrm{H}$ atoms in $\mathrm{I}-43 d \mathrm{NbH}_{3}$ is $1.34 \AA$ at $200 \mathrm{GPa}\left(V_{0} / V=1.69\right)$, which is actually a little longer than the second shortest separation of $1.2 \AA$ in pure solid hydrogen ${ }^{48,57,58}$ at the same pressure. However, this separation is considerably in excess of the shortest $\mathrm{H}-\mathrm{H}$ distance of $0.75 \AA$ in pure solid hydrogen at $1 \mathrm{~atm}, 0.87 \AA$ in $\mathrm{GeH}_{4}$ at $220 \mathrm{GPa}^{7}$ and $0.79 \AA$ in $\mathrm{SnH}_{4}$ at $120 \mathrm{GPa}^{8}{ }^{8}$ which, interestingly, suggests that there are no semi-molecular paired hydrogens in the highpressure structure of $\mathrm{NbH}_{3}$. We will return to a comparative 


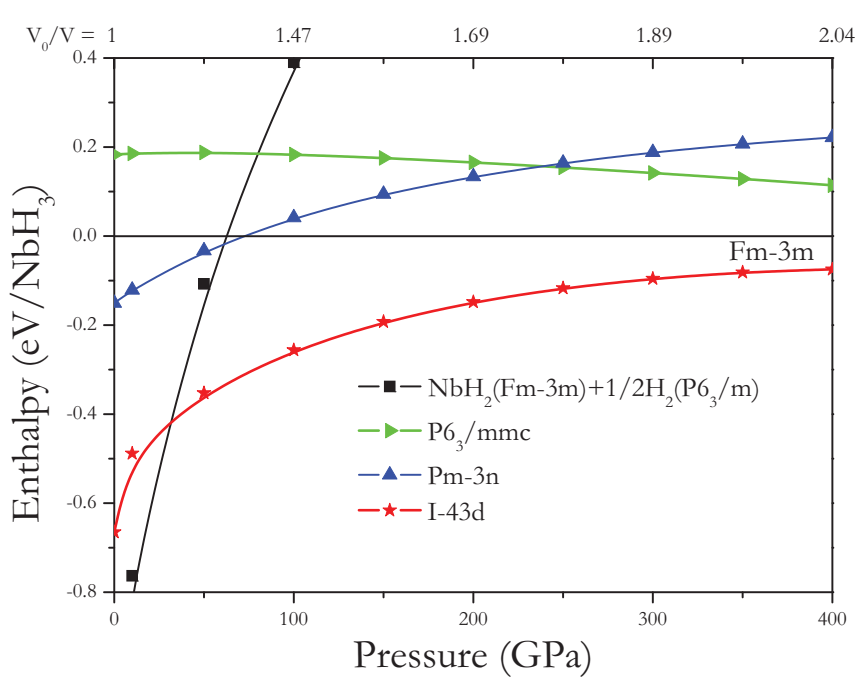

FIG. 8. (Color online) Ground-state static enthalpy curves per formula unit as a function of pressure with respect to the $F m-3 m$ structure for static $\mathrm{NbH}_{3}$. The decomposition enthalpies of $\mathrm{NbH}_{3}$ to $\mathrm{NbH}_{2}+\mathrm{H}_{2}$ are also presented. We adopted the $\mathrm{Fm}-3 \mathrm{~m}$ and $\mathrm{P}_{3} / \mathrm{m}$ structures for $\mathrm{NbH}_{2}$ and $\mathrm{H}_{2}$, respectively. The relative compression $\left(V_{0} / V\right)$ is again indicated.

analysis of the $\mathrm{H}-\mathrm{H}(\mathrm{Nb}-\mathrm{H}$ and $\mathrm{Nb}-\mathrm{Nb})$ separations in the discussion of $\mathrm{NbH}_{\mathrm{n}}$ below.

\section{E. $\mathrm{NbH}_{4}$}

We find only a single structure for static $\mathrm{NbH}_{4}$, which is stable solely at high pressures. The predicted structure for $\mathrm{NbH}_{4}$ is a body-centered tetragonal $I 4 / \mathrm{mmm}(Z=2$, Pearson symbol $t I 10)$ geometry with a slightly distorted bcc $\mathrm{Nb}$ metal lattice, the $\mathrm{H}$ atoms fully occupying the $T$ interstices on both of the top and bottom faces, and only two of the $T$ interstices on four lateral sides; this is shown in Fig. 9.

Interestingly, the recently predicted high pressure structure for $\mathrm{CaH}_{6}{ }^{5}$ shows that the $\mathrm{H}$ atoms occupy all of the $T$ interstices of the bcc $\mathrm{Ca}$ metal lattice. Static ground-state enthalpy calculations (see Fig. 10) here now indicate that $\mathrm{NbH}_{4}$ will dissociate to $\mathrm{NbH}_{2}$ and $\mathrm{H}_{2}$ at $33 \mathrm{GPa}$, then to $\mathrm{NbH}_{3}$ and $\mathrm{H}_{2}$ between 33-287 GPa, above which it will be stable in the I $4 / \mathrm{mmm}$ structure.

The shortest separation between two $\mathrm{H}$ atoms in $I 4 / \mathrm{mmm}$ $\mathrm{NbH}_{4}$ is $1.21 \AA$ at $300 \mathrm{GPa}\left(V_{0} / V=1.86\right)$. This occurs in the four lateral sides of the unit cell; two of these are clearly visible in Fig. 9. At such high pressures (300 GPa), all hydrogens

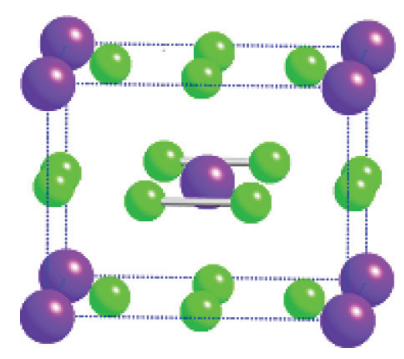

FIG. 9. (Color online) Predicted static high-pressure ground-state I4/mmm structures for $\mathrm{NbH}_{4}$. The two horizontal lines show the two nearest $\mathrm{H}$ atoms.

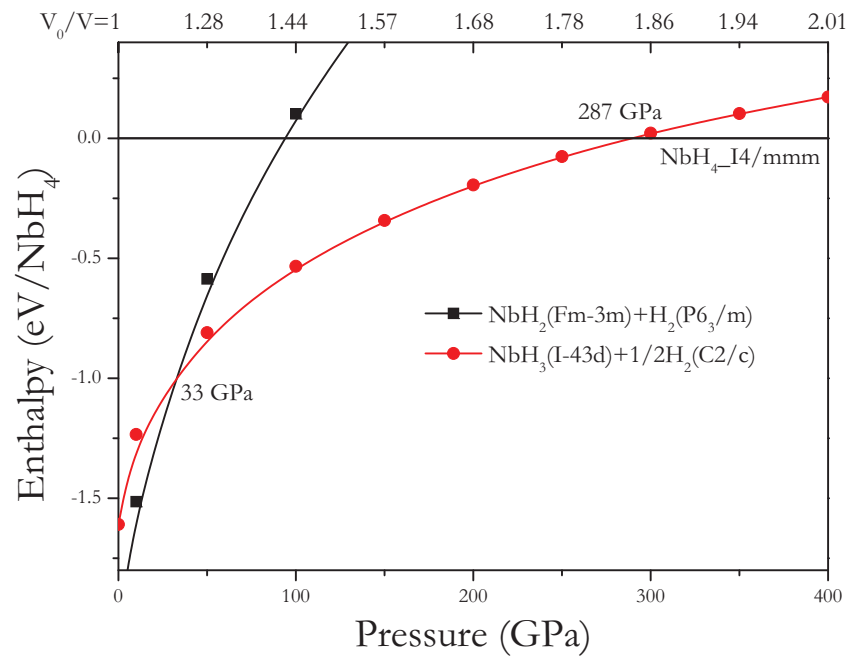

FIG. 10. (Color online) Ground-state static enthalpy curves per formula unit for static $\mathrm{NbH}_{4}$ as a function of pressure with respect to the $14 / \mathrm{mmm}$ structure. The decomposition enthalpies of $\mathrm{NbH}_{4}$ into $\mathrm{NbH}_{2}+\mathrm{H}_{2}$ and $\mathrm{NbH}_{3}+\mathrm{H}_{2}$ are also presented. We have considered the Fm-3m structure for $\mathrm{NbH}_{2}, I-43 d$ for $\mathrm{NbH}_{3}$, and $\mathrm{PG}_{3} / m$ and $C 2 / c$ for $\mathrm{H}_{2}$. The relative compression $\left(V_{0} / V\right)$ is also indicated at the top of the figure.

then have no choice but to approach each other, and the identification of a "bond" is then to some extent arbitrary. A calibration might be obtained from the optimum next nearest $\mathrm{H}-\mathrm{H}$ separation in hydrogen itself under pressure (where it still appears paired, and with a nearest $\mathrm{H}-\mathrm{H}$ separation of $0.75 \AA$ ) of $1.2 \AA$ at $200 \mathrm{GPa}^{57}$ Returning to $\mathrm{NbH}_{4}$ at $300 \mathrm{GPa}$, the $\mathrm{H}-\mathrm{H}$ separation in the top and bottom faces is $1.58 \AA$, while the $\mathrm{H}-\mathrm{H}$ separation between atoms in top or bottom and the sides is $1.59 \AA$. These $\mathrm{H}-\mathrm{H}$ separations are also in excess of those for pure solid hydrogen and in the high pressure structures of $\mathrm{GeH}_{4}$ and $\mathrm{SnH}_{4}$, so that all in all, we arrive at the conclusion that there are no semimolecular paired hydrogen units in the high-pressure structure of $\mathrm{NbH}_{4}$.

\section{F. $\mathrm{NbH}_{5}$}

When the number of hydrogen atoms per niobium reaches five, an extensive search indicates that all the static groundstate structures calculated for this composition are unstable with respect to decomposition into $\mathrm{NbH}_{2}+\mathrm{H}_{2}, \mathrm{NbH}_{3}+$ $\mathrm{H}_{2}$, and $\mathrm{NbH}_{4}+\mathrm{H}_{2}$ up to at least $500 \mathrm{GPa}$ (see Fig. S4 in Ref. 17). The predicted $P-1$ and $C 2 / m$ structures are illustrated in Fig. S5 in Ref. 17. There are no $\mathrm{H}_{2}$ pairs in the high-pressure structures of $\mathrm{NbH}_{5}$.

\section{G. $\mathrm{NbH}_{6}$}

Two structures compete in our static ground-state calculations for the high pressure phases of this stoichiometry. One is an orthorhombic Cmmm ( $Z=2$, Pearson symbol $o C 14)$ geometry, and the other is $C 2 / c(Z=8$, Pearson $m C 56)$. As Fig. 11 shows, these structures are both unstable with respect to decomposition into $\mathrm{NbH}_{2}$ or $\mathrm{NbH}_{3}$ for pressures lower than $287 \mathrm{GPa}\left(V_{0} / V=1.87\right)$. From this pressure to $\sim 400 \mathrm{GPa}$, static $C 2 / c-\mathrm{NbH}_{6}$ is stable (and metallic, see below) but its enthalpy 


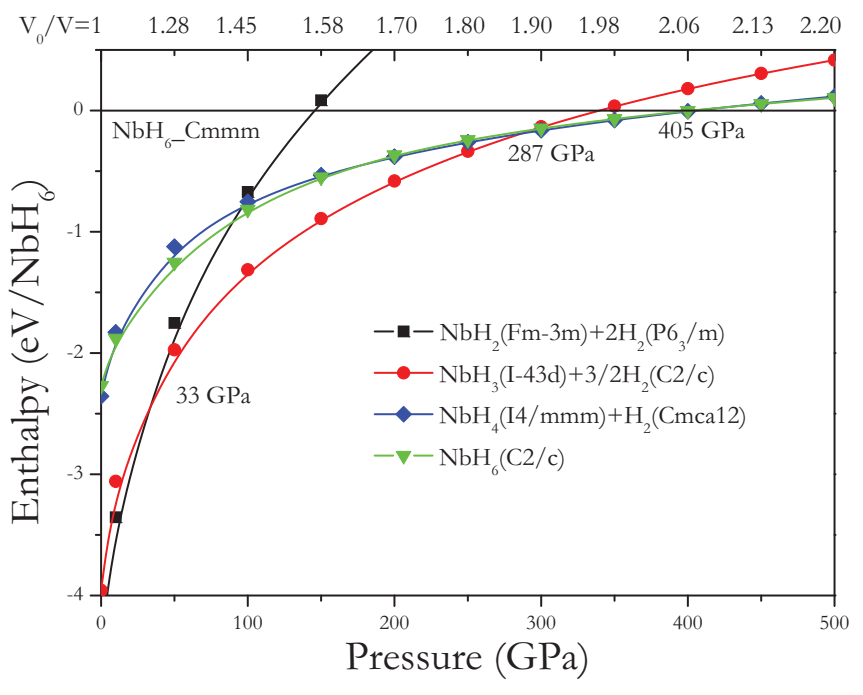

FIG. 11. (Color online) Static ground-state enthalpy curves per formula unit for $\mathrm{NbH}_{6}$ as a function of pressure; the reference line is the $\mathrm{Cmmm}$ structure (stable at highest pressures). The enthalpies of decompositions of $\mathrm{NbH}_{6}$ into $\mathrm{NbH}_{2}+\mathrm{H}_{2}, \mathrm{NbH}_{3}+\mathrm{H}_{2}$, and $\mathrm{NbH}_{4}$ $+\mathrm{H}_{2}$ are also presented. We have considered the $\mathrm{Fm}-3 \mathrm{~m}$ structure for $\mathrm{NbH}_{2}, I-43 d$ for $\mathrm{NbH}_{3}, I 4 / \mathrm{mmm}$ for $\mathrm{NbH}_{4}$, and $\mathrm{PG}_{3} / \mathrm{m}, \mathrm{C2} / \mathrm{c}$, and Cmcal 2 for $\mathrm{H}_{2}$. The relative compression $\left(V_{0} / V\right)$ is also shown.

is very close to that of $\mathrm{NbH}_{4}+\mathrm{H}_{2}$. Above $\sim 400 \mathrm{GPa}$, which is the highest pressure studied, the $C m m m-\mathrm{NbH}_{6}$ structure is stable with respect to all decompositions.

The two high-pressure $\mathrm{NbH}_{6}$ structures, $\mathrm{Cmmm}$ and $C 2 / \mathrm{c}$ are quite different, however. Each ground-state structure features a distinct hydrogen sublattice. To discuss these we display a histogram of $\mathrm{H}-\mathrm{H}$ separations for the two lattices (see Fig. S6),${ }^{17}$ both computed in their regions of stability, at $400 \mathrm{GPa}\left(V_{0} / V=2.06\right.$, see Fig. 11).

In both structures, we therefore have a quite continuous dispersal of short H-H separations, and it is immediately apparent that there is no unambiguous way to choose a sensible cutoff for $\mathrm{H}-\mathrm{H}$ separations, a value below which it would be agreed that bonding should exist. In $\mathrm{H}_{2}$ itself, at the same pressure the shortest $\mathrm{H}-\mathrm{H}$ separation is still close to molecular, at $0.78 \AA$, while the next nearest separation is $1.06 \AA .{ }^{57}$ If we choose, somewhat arbitrarily, $1.42 \AA$ (depicted by the arrows in Fig. S6) ${ }^{17}$ as a cutoff for identifying $\mathrm{H}-\mathrm{H}$ separations in $\mathrm{Cmmm}$ $\mathrm{NbH}_{6}$, we then obtain the clathrate-type structure shown in two views in Fig. 12.

The monoclinic $C 2 / c(Z=4$, Pearson symbol $m C 56) \mathrm{NbH}_{6}$ structure presents similar problems with respect to extracting $\mathrm{H}-\mathrm{H}$ coordinations, as the bottom of Fig. S6 shows. ${ }^{17}$ If we again take $1.42 \AA$ as the separation cutoff, this structure may be viewed as composed of layered-chainlike networks of hydrogens, with niobiums dispersed around the periphery (see Fig. 13).

As the histogram of Fig. S6 shows, ${ }^{17}$ the closest groundstate separation between two $\mathrm{H}$ atoms in static $C m m m \mathrm{NbH}_{6}$ is $1.00 \AA$ at $400 \mathrm{GPa}$ (between the two atoms parallel to $x$ axis). In the other high-pressure $\mathrm{NbH}_{6}$ form, $C 2 / c$, the shortest $\mathrm{H}-\mathrm{H}$ separation at $400 \mathrm{GPa}$ is also $1.00 \AA$. There is again no clear pairing of $\mathrm{H}$ atoms in either structure. By comparison the networks of hydrogens, all the hydrogens in the
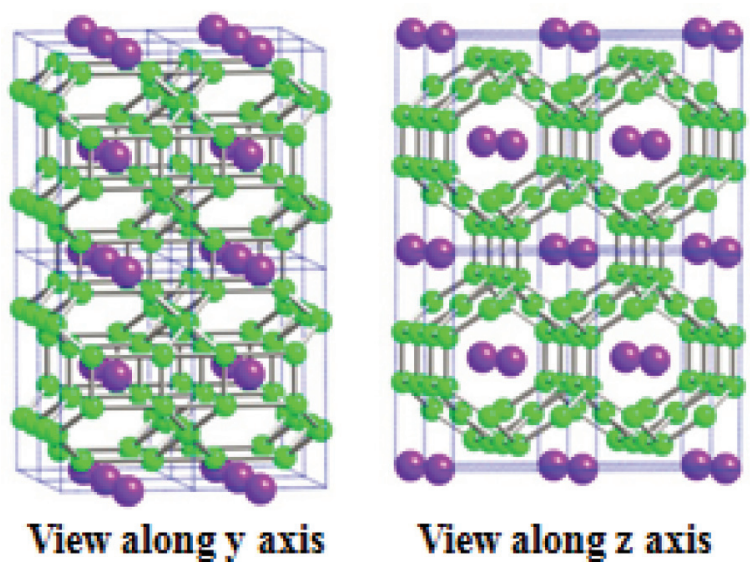

FIG. 12. (Color online) Predicted static high-pressure groundstate Cmmm structure for $\mathrm{NbH}_{6}$ at $400 \mathrm{GPa}$. The lines indicate $\mathrm{H}-\mathrm{H}$ separations shorter than $1.42 \AA$.

high-pressure structure $(\mathrm{Im}-3 \mathrm{~m})$ of $\mathrm{CaH}_{6}$ form a sodalite like clathrate and the $\mathrm{H}-\mathrm{H}$ separations between $\mathrm{H}-\mathrm{H}$ neighbors are all $1.24 \AA$ at a much lower pressure, $150 \mathrm{GPa} .{ }^{5}$ The important difference between the $\mathrm{CaH}_{6}$ cagelike structure and our $\mathrm{NbH}_{6}$ equivalent is that our structure is not stabilized until much higher pressures (than the $150 \mathrm{GPa}$ reported for $\mathrm{CaH}_{6}$ ). The relative compressions are 2.06 for $\mathrm{NbH}_{6}$ at $400 \mathrm{GPa}$ and 1.85 for $\mathrm{CaH}_{6}$ at $150 \mathrm{GPa}$.

In order to make clear in electronic terms the reasons for concentrating above on $\mathrm{H}-\mathrm{H}$ bonding, especially in $\mathrm{NbH}_{6}$, we and others have found in alkali metal, alkaline-earth metal hydrides, and also in $\mathrm{WH}_{n}$, that a way to understand the structure is to think of initial electron transfer from the electropositive element to $\mathrm{H}_{2}$ units. With sufficient electron transfer, an $\mathrm{H}_{2}{ }^{2 \delta-}$ will be expected to dissociate into $2 \mathrm{H}^{\delta-}$, which would then enter into an extended hydridic network. The thought process here is stepwise; in reality, effective partial electron transfer and $\mathrm{H}_{2}$ dissociation would be happening concurrently in the formation of the phase. $\mathrm{A} \mathrm{Nb}$ atom has five valence electrons, a number insufficient to reduce $3 \mathrm{H}_{2}$ to $6 \mathrm{H}^{-}$, so one could imagine some $\mathrm{H}_{2}$ molecules remaining as bonded pairs, even if the $\mathrm{H}-\mathrm{H}$ bonding is weakened, but we do not find this.

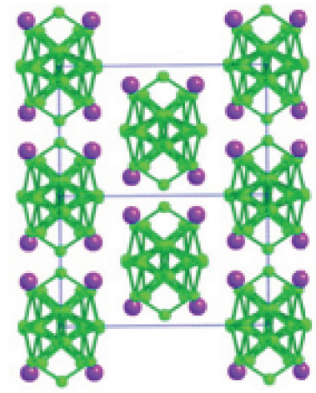

View along $\mathrm{z}$ axis

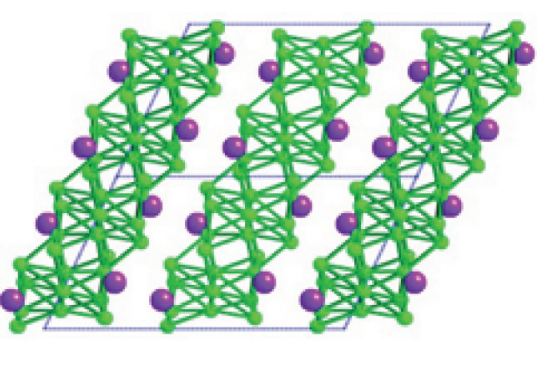

View along $y$ axis
FIG. 13. (Color online) Predicted static high-pressure groundstate $C 2 / c$ structures at $400 \mathrm{GPa}$ for $\mathrm{NbH}_{6}$. H-H separations shorter than $1.42 \AA$ are again drawn as lines. 


\section{H. $\mathrm{NbH}_{12}$}

This very high hydride is discussed (as mentioned above) in a separate paper, which also includes $\mathrm{NbH}_{24}$, and $\mathrm{NbH}_{48}$, but is briefly mentioned here with respect to the physical and chemical expectations of the eventual appearance of hydrogen pairs, a matter also raised in the foregoing. In our study ${ }^{9}$ of the ground-state tungsten hydrides, there were no hydrogen pairs when the number of hydrogen atoms in $\mathrm{WH}_{\mathrm{n}}$ fell below six. However, we found $\mathrm{H}$ pairs in $\mathrm{WH}_{8} .{ }^{9}$ Accordingly, we examined in considerable detail the geometrical and electronic structure of niobium hydrides with even higher concentrations of hydrogen, and indeed we find molecular units in the high-pressure structures of $\mathrm{NbH}_{12}$, with unusual intermediate $\mathrm{H}-\mathrm{H}$ separations. However, none of these compositions lie on the convex hull (see below). Their electronic structures are particularly interesting at higher pressures, however, and (again as indicated earlier) these will be discussed in detail in a further paper.

\section{Enthalpical stability for different stoichiometries under pressure}

Let us now assemble the enthalpy relationships for the $\mathrm{NbH}_{\mathrm{n}}$ series in the form of tie-line graphs, each in the standard convex hull of comparative stability (see Fig. 14). The reference level is pure niobium and pure hydrogen, each in its most stable form at the pressure specified. As can be seen, at $1 \mathrm{~atm}$, the lower hydrides $\mathrm{Nb}_{4} \mathrm{H}_{3}, \mathrm{NbH}, \mathrm{NbH}_{2}, \mathrm{NbH}_{3}$, and $\mathrm{NbH}_{4}$ are all stable with respect to disproportionation into the elements, but $\mathrm{NbH}_{3}$ and $\mathrm{NbH}_{4}$ are only metastable, and are predicted to decompose into $\mathrm{Nb}_{4} \mathrm{H}_{3}$ and $\mathrm{H}_{2}, \mathrm{NbH}$ and $\mathrm{H}_{2}$ or $\mathrm{NbH}_{2}$ and $\mathrm{H}_{2}$.

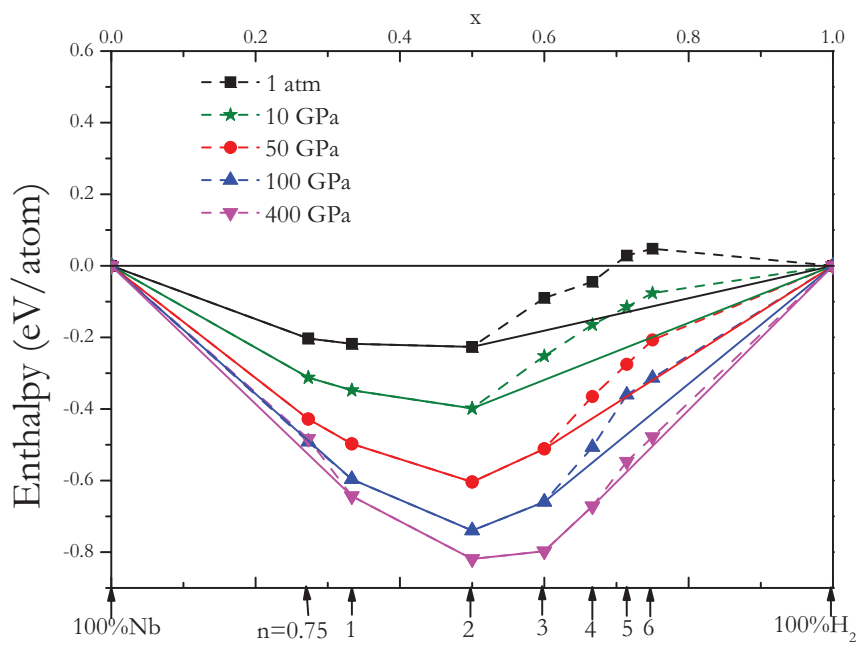

FIG. 14. (Color online) Ground-state and static enthalpy of formation per atom of the $\mathrm{Nb}_{(1-x)}\left(\mathrm{H}_{2}\right)_{x}$ phases with respect to their separated counterparts; hydrogen molar content $(x=0$ corresponds to pure niobium; $x=1$ to pure hydrogen) for the ground state and $P=1 \mathrm{~atm}, 10,50,100$, and $400 \mathrm{GPa}$. The stoichiometric index $n$ (in $\mathrm{NbH}_{n}$ ) is indicated at the bottom. The symbols on the solid line indicate that the hydride is stable at the corresponding pressure, while those on the dashed line represent the fact that the hydrides are unstable with respect to decomposition or disproportionation into other hydrides and niobium or hydrogen.
Indeed, $\mathrm{NbH}_{x}\left(x<1\right.$, and off stoichiometry) and $\mathrm{NbH}_{2}$ are known. As the pressure increases, the formation enthalpy of each hydride becomes more and more negative. At $10 \mathrm{GPa}$, the formation enthalpy of all the hydrides studied becomes negative, and at $50 \mathrm{GPa} \mathrm{NbH}_{3}$ falls on the convex hull. Note that $\mathrm{NbH}_{4}$ becomes stable at a pressure of $\sim 400 \mathrm{GPa}$, and it is also possible that $\mathrm{NbH}_{6}$ (and perhaps $\mathrm{NbH}_{5}$ ) becomes stable at still higher pressure. In the ground state, pressure clearly favors the formation of $\mathrm{Nb}$ hydrides with high content of hydrogen, even while $\mathrm{NbH}_{2}$ remains the most stable hydride over the whole pressure range studied.

\section{J. The relative enthalpy stability of different hydrides including dynamical contributions}

We turn now to dynamic contributions to the enthalpy. Because of the very light mass of the hydrogen atom, quantum effects are expected to be a priori important and the hydrogen zero-point energy (ZPE) may well be large enough to affect the structural stability range of the computed phases. ${ }^{48}$ Using zone summation we estimated the ZPEs for each $\mathrm{Nb}$ hydride, pure $\mathrm{H}_{2}$ and $\mathrm{Nb}$ under pressure using $E_{\mathrm{ZPE}}=\frac{1}{2} \sum_{\mathrm{q}, \mathrm{j}} \hbar \omega_{j}(\boldsymbol{q})$ within the harmonic approximation, where $j$ indicates a phonon branch at wave vector $\boldsymbol{q}$; $\omega_{\mathrm{j}}(\boldsymbol{q})$ is the frequency at wave vector $\boldsymbol{q}$ and calculated using the PHONOPY code ${ }^{40}$ the results are summarized in Table I.

The effects on ground-state phase transitions are best seen graphically (see Fig. 15). The results presented are obtained on the assumption that the total enthalpy is the sum of the static system and the contribution associated with the phonons determined in a harmonic approximation (eventually a self-consistent approach to this may be required). $\mathrm{NbH}_{4}$ has a smaller $\mathrm{ZPE}$ of $0.153 \mathrm{eV} / \mathrm{NbH}_{4}$ when compared with the corresponding sum of $\mathrm{NbH}_{3}$ and $1 / 2 \mathrm{H}_{2} \mathrm{ZPEs}$, indicating

TABLE I. Calculated zero-point energies per atom for different niobium hydrides and niobium and hydrogen at 1 atm and under pressure.

\begin{tabular}{lccc}
\hline \hline Systems & Space group & $\begin{array}{c}\text { Pressure } \\
(\mathrm{GPa})\end{array}$ & $\begin{array}{c}\text { Zero-point } \\
\text { energies (eV/atom) }\end{array}$ \\
\hline $\mathrm{NbH}$ & Cccm & 1 atm & 0.1285 \\
& $R-3 m$ & 50 & 0.1265 \\
& & 300 & 0.196 \\
$\mathrm{NbH}_{2}$ & Pnma & 50 & 0.1703 \\
& & 300 & 0.252 \\
$\mathrm{NbH}_{3}$ & $I-43 d$ & 50 & 0.1878 \\
& & 300 & 0.2728 \\
$\mathrm{NbH}_{4}$ & $I 4 / m m m$ & 300 & 0.2498 \\
& & 400 & 0.274 \\
$\mathrm{NbH}_{6}$ & $C m m m$ & 400 & 0.2769 \\
$\mathrm{H}_{2}$ & $P 66_{3} / m$ & 1 atm & 0.129 \\
& & 50 & 0.241 \\
& $C 2 / c$ & 300 & 0.311 \\
& $C m c a-12$ & 400 & 0.321 \\
$\mathrm{Nb}$ & $I m-3 m$ & 1 atm & 0.025 \\
& & 50 & 0.0381 \\
& & 300 & 0.0638 \\
& & 400 & 0.071 \\
\hline \hline
\end{tabular}



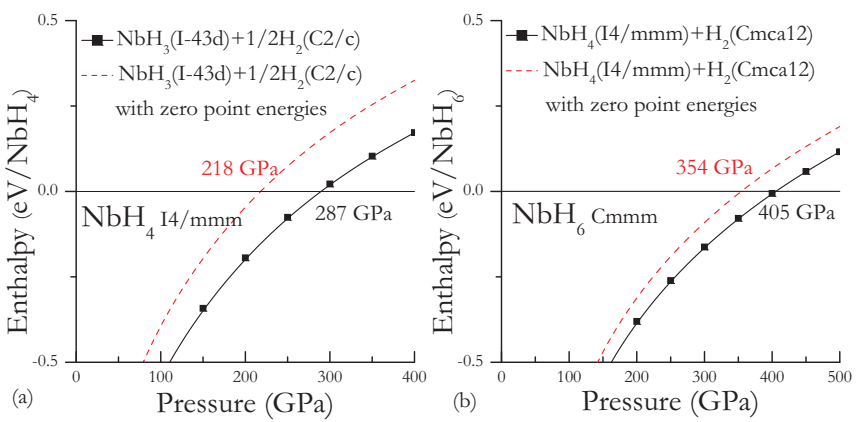

FIG. 15. (Color online) Ground-state enthalpy curves per formula unit (with and without zero-point energies) for the most stable $\mathrm{NbH}_{4}$ (a) and $\mathrm{NbH}_{6}$ (b) phases with respect to $\mathrm{NbH}_{3}+\mathrm{H}_{2}$ and $\mathrm{NbH}_{4}+$ $\mathrm{H}_{2}$, respectively. The ZPE differences are taken to be approximately pressure independent.

that after inclusion of ZPE [see Fig. 15(a)], $\mathrm{NbH}_{4}$ may become stable at a much lower pressure, $\sim 218 \mathrm{GPa}$, than that indicated in Fig. 10. $\mathrm{NbH}_{6}$ has also a smaller $\mathrm{ZPE}$ of $0.074 \mathrm{eV} / \mathrm{NbH}_{6}$ compared with the sum of ZPEs for $\mathrm{NbH}_{4}$ and $\mathrm{H}_{2}$, so $\mathrm{NbH}_{6}$ is also expected to become more stable at a lower pressure of $\sim 354 \mathrm{GPa}$ when ZPE is included [see Fig. 15(b)]. Therefore inclusion of the ZPE in the ground state may reduce the stability range of $\mathrm{NbH}_{3}$, while $\mathrm{NbH}_{4}$ and $\mathrm{NbH}_{6}$ will actually become stable at lower pressure.

\section{K. Dynamical stability for different stoichiometries}

We have also studied the dynamical stability of various structures for $\mathrm{NbH}_{n}(n=1-4,6)$ by calculating their phonon dispersion curves using a supercell method. It is found that except for $\mathrm{NbH}_{5}$ all the predicted structures of each hydride studied are dynamically stable (see Figs. S7 and 8); ${ }^{17}$ there are no imaginary frequencies at the level of harmonic theory.

The calculated phonon frequencies of different structures in niobium hydrides separate into two groups: low-frequency (mostly associated to $\mathrm{Nb}$ atoms) and high-frequency modes, those dominated by light $\mathrm{H}$ atoms. The gap between the lowand high-frequency modes gradually decreases as the content of hydrogen increases, and, as expected, the highest frequencies also increase with increasing pressure. The absence of really high frequencies corresponding to the intramolecular vibrations of $\mathrm{H}_{2}$ molecules supports our argument above (this based on separations) for the absence of paired hydrogens in the structures of the niobium hydrides studied here.

\section{Electronic properties}

Figure 16 presents the calculated electronic density of states (DOS) per valence electron for static Cccm-NbH, Fm$3 m-\mathrm{NbH}_{2}, I-43 d-\mathrm{NbH}_{3}, I 4 / m m m-\mathrm{NbH}_{4}$, and $C m m m-\mathrm{NbH}_{6}$, each at a pressure corresponding to its stability region. The corresponding band structures are given in Ref. 17, where we also compare the five stoichiometries at a single pressure, namely, $50 \mathrm{GPa}$. In Fig. 16, the width of the valence band increases as $n$ in $\mathrm{NbH}_{n}$ : this is the expected outcome of the pressure induced increase in average density. All of the hydrides are predicted to be metallic, which is important to the early motivating arguments relating to potential hydride

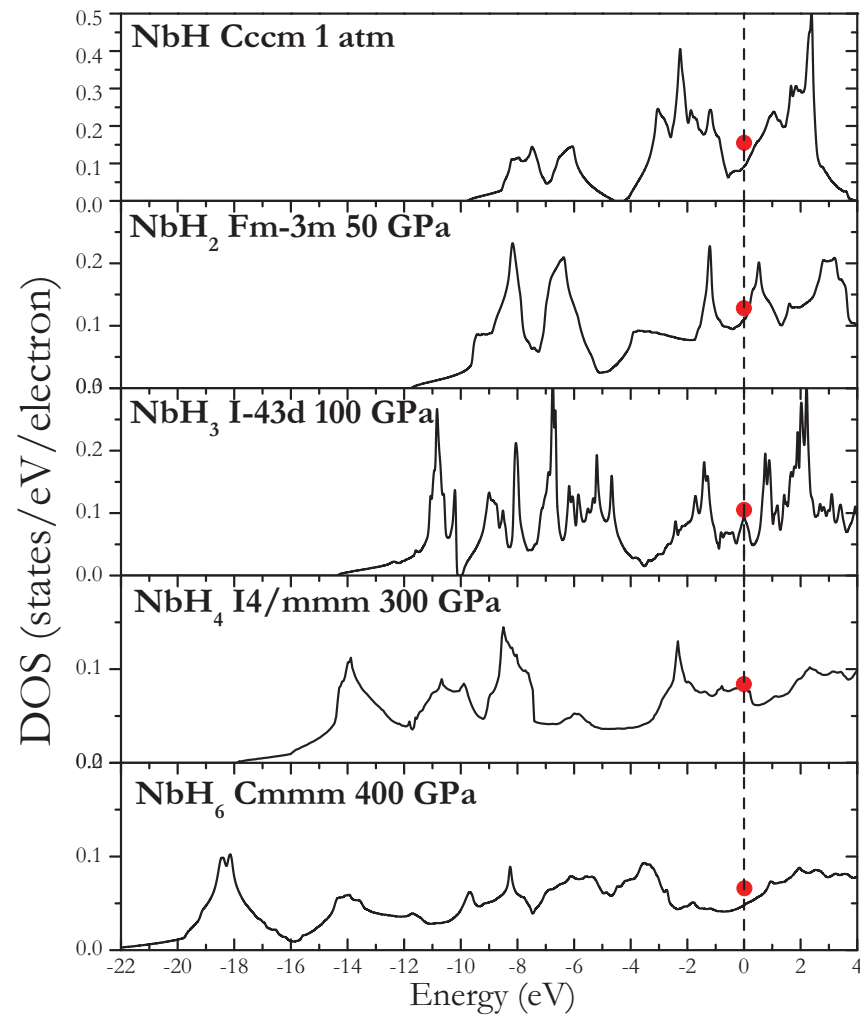

FIG. 16. (Color online) Density of states (DOS) per valence electron of $\mathrm{NbH}, \mathrm{NbH}_{2}, \mathrm{NbH}_{3}, \mathrm{NbH}_{4}$, and $\mathrm{NbH}_{6}$ at 1atm, 50, 100, 300 , and $400 \mathrm{GPa}$, respectively. The red dots present the DOS for equivalent free-electron systems. It is clear that all of these hydrides are metallic.

superconductivity. An analysis of the contributions of various atomic orbital symmetries to the total electronic DOS (see again Ref. 17) indicates that the electronic DOS at the Fermi level originates, as expected, mostly from the $\mathrm{Nb} 4 d$ orbitals. However, in the higher hydrides, given the polarity $\mathrm{Nb}^{\delta+} \mathrm{H}^{\delta-}$, one also expects and obtains contributions to the DOS near the Fermi level from the hydrogens as well, especially for $\mathrm{NbH}_{4}$ and $\mathrm{NbH}_{6}$. The hydrides studied have reasonably high electronic densities of states at the Fermi level, as one can judge through comparison with the DOS obtained in a free electron approximation (the red dot in each DOS). The Fermi level in general does not fall in a pseudogap, as it often does for binaries.

Turning back to one of the motivating factors of this study, we attempted through standard estimations to assess the occurrence of possible superconductivity in these hydrides. This proved possible only in $\mathrm{NbH}_{2}, \mathrm{NbH}$, and $\mathrm{NbH}_{4}$, as the other hydrides are predicted to crystallize in unit cells that to date are too large for us to undertake at this time a full analysis of the electron-phonon coupling. For $\mathrm{NbH}_{2}$, the calculated electron-phonon coupling parameter $\lambda$ (see the Computational Details for its definition) for the $F m-3 m$ structure at $1 \mathrm{~atm}$ is only 0.5 and the logarithmic average frequency $\omega_{\log }$ is equivalent to $247 \mathrm{~K}$. Applying the Allen and Dynes modified McMillan equation ${ }^{45}$ with a typical choice of Coulomb pseudopotential $\mu^{*}$ of 0.13 , the estimated $T_{c}$ is then less than $2 \mathrm{~K}$; this is quite consistent with experimental 
TABLE II. Calculated phonon frequency logarithmic averages $\left(\omega_{\log }\right)$, electron-phonon coupling parameters $(\lambda)$, and critical temperatures $\left(T_{c}\right)$ for $\mathrm{NbH}, \mathrm{NbH}_{2}$, and $\mathrm{NbH}_{4}$ at different pressures.

\begin{tabular}{lcccccc}
\hline \hline Hydride & Structure & $\mathrm{P}(\mathrm{GPa})$ & Lambda $(\lambda)$ & $\omega_{\log }(\mathrm{K})$ & $T_{c}\left(\mathrm{~K}, \mu^{*}=0.1\right)$ & $T_{c}\left(\mathrm{~K}, \mu^{*}=0.13\right)$ \\
\hline $\mathrm{NbH}$ & $C c c m$ & $1 \mathrm{~atm}$ & 0.51 & 188 & 2.4 & 1.5 \\
$\mathrm{NbH}_{2}$ & $F m-3 m$ & $1 \mathrm{~atm}$ & 0.48 & 251 & 2.6 & 1.5 \\
& & $50 \mathrm{GPa}$ & 0.41 & 303 & 1.5 & 0.7 \\
$\mathrm{NbH}_{4}$ & $I 4 / \mathrm{mmm}$ & $300 \mathrm{GPa}$ & 0.82 & 938 & 47 & 38 \\
\hline \hline
\end{tabular}

observations. Although $\mathrm{NbH}_{2}$ has an impressive density of states at the Fermi level (see Fig. 16), the weak electronphonon coupling parameter $\lambda$ and small logarithmic average frequency $\omega_{\log }$, attributed to an elongated separation between $\mathrm{Nb}$ and $\mathrm{H}(2.0 \AA)$, and a very long separation between $\mathrm{H}$ and $\mathrm{H}(2.3 \AA)$, ultimately lead to the very low $T_{c}$. As pressure increases, $\omega_{\log }$ increases, $\lambda$ decreases and finally the calculated $T_{c}$ decreases to about $1 \mathrm{~K}$ at $50 \mathrm{GPa}$, see Table II. $\mathrm{NbH}_{2}$ is therefore not expected to be a significant superconductor at $\mathrm{P}=1 \mathrm{~atm}$, and according to our calculations, this will not change at higher pressures.

For $\mathrm{NbH}$, the estimated $T_{c}$ of $1.5 \mathrm{~K}$ (assuming $\mu^{*}=0.13$ ) at $1 \mathrm{~atm}$ is similar to that of $\mathrm{NbH}_{2}$, indicating that $\mathrm{NbH}$ is also not a promising candidate for superconductivity. For $\mathrm{NbH}_{4}$, however, the situation is markedly different; a relatively high $T_{c}$ of $38 \mathrm{~K}$ (again taking $\mu^{*}=0.13$ ) is estimated at $300 \mathrm{GPa}$. This can be attributed to a strong electron-phonon coupling parameter $\lambda$ of 0.82 , and to a large logarithmic average frequency $\omega_{\log }$ of $938 \mathrm{~K}$, reflecting the increased presence of additional hydrogen. Further analysis of the Eliashberg spectral function and phonon DOS demonstrates that the high frequencies, mainly associated with hydrogen vibrations, play a quite significant role in creating a relatively strong electron-phonon coupling parameter $\lambda$ (see Fig. S14). ${ }^{17}$

How realistic is the prospect of observing relatively high $T_{c}$ superconductivity in compressed $\mathrm{NbH}_{4}$ ? The first question is whether we can trust the $T_{c}$ calculations. To answer this question, we point to some previous experience: Using the Allen-Dynes modified McMillan equation, the estimated $T_{c}$ for numerous materials are reasonably consistent with experimental measurements. For example, by using values of $\mu^{*}$ from 0.05 to 0.15 , the $T_{c}$ values at $60 \mathrm{GPa}$ for calcium estimated using the Allen-Dynes modified McMillan equation are from 6.8 to $2.2 \mathrm{~K}$, in agreement with the measured $T_{c}$ of 2.5 and $5 \mathrm{~K}$ at the same pressure. ${ }^{60}$ Using $\mu^{*}=0.1$, the estimated $T_{c}=19 \mathrm{~K}^{61}$ at high pressure for lithium is in good agreement with the experimental value of $20 \mathrm{~K}^{62}$ Very reasonable superconducting temperatures as compared with experiment are obtained throughout the stable pressure range of $\mathrm{Si}(\mathrm{V})$ from 18 to $35 \mathrm{GPa}$ with $\mu^{*}=0.12$. $^{63}$

The estimate of $T_{c}$ for $I 4 / \mathrm{mmm}-\mathrm{NbH}_{4}$ at $300 \mathrm{GPa}$ is $38-47 \mathrm{~K}$ when we use values of $\mu^{*}$ from 0.13 to 0.1 . Although the I4/mmm structure for $\mathrm{NbH}_{4}$ is enthalpically stable above 285 $\mathrm{GPa}$, when zero-point energies are considered as well, it can be stabilized at a pressure as low as $215 \mathrm{GPa}$, which puts it into a pressure regime that is achievable experimentally, although we realize it is still not easy to do. Moreover, according to our calculated pressure effects on superconductivity of $\mathrm{NbH}_{2}$ and many other hydrides $\left(\mathrm{GaH}_{3}{ }^{64}\right.$ and $\left.\mathrm{CaH}_{6}{ }^{5}\right), \mathrm{NbH}_{4}$ should have a $T_{c}$ higher than $38 \mathrm{~K}$ at a pressure lower than $300 \mathrm{GPa}$. We therefore think that it is a realistic experimental prospect that $\mathrm{NbH}_{4}$ can be made at high pressures and have a $T_{c}$ in excess of $38 \mathrm{~K}$.

\section{Interatomic separations and coordination number as $n$ varies in $\mathbf{N b H}_{\mathbf{n}}$}

Hydrogen atoms/ions that are introduced must interact with or be bonded to the niobium ions. The geometry and strength (as measured by the $\mathrm{Nb}-\mathrm{H}$ separations) of these bonding interactions is of considerable interest, as is the effect of the introduced hydrogen on the $\mathrm{Nb}-\mathrm{Nb}$ bonding. This section addresses these questions.

In Fig. 17, we show a histogram of $\mathrm{Nb}-\mathrm{H}$ and $\mathrm{Nb}-\mathrm{Nb}$ separations in the ground-state $\mathrm{NbH}_{n}$ phases $(n=1-4$, and $6)$, each at a pressure for which the phase is stable. This means a pressure range from 1 atm for $\mathrm{NbH}$ to $400 \mathrm{GPa}_{\text {for }} \mathrm{NbH}_{6}$. We also gain some insight from comparing the various hydrides at one and the same pressure-this is presented in Fig. S15 in Ref. 17.

As Fig. 17 clearly shows, the electronic structures are such that $\mathrm{Nb}-\mathrm{H}$ separations are not affected much by the hydrogen content of the associated niobium hydride at least for static arrangements. The shortest $\mathrm{Nb}-\mathrm{H}$ separation changes from 1.68 to $1.94 \AA$ in $\mathrm{NbH}_{n}$, as $\mathrm{n}$ ranges from 1 to 4 and 6. For comparison, the calculated $\mathrm{Nb}-\mathrm{H}$ separations in molecular hydrides $\mathrm{NbH}_{2}$ and $\mathrm{NbH}_{4}$ at 1 atm are 1.79 and $1.7 \AA$, respectively. ${ }^{15}$ The $\mathrm{Nb}-\mathrm{H}$ elongation in the extended

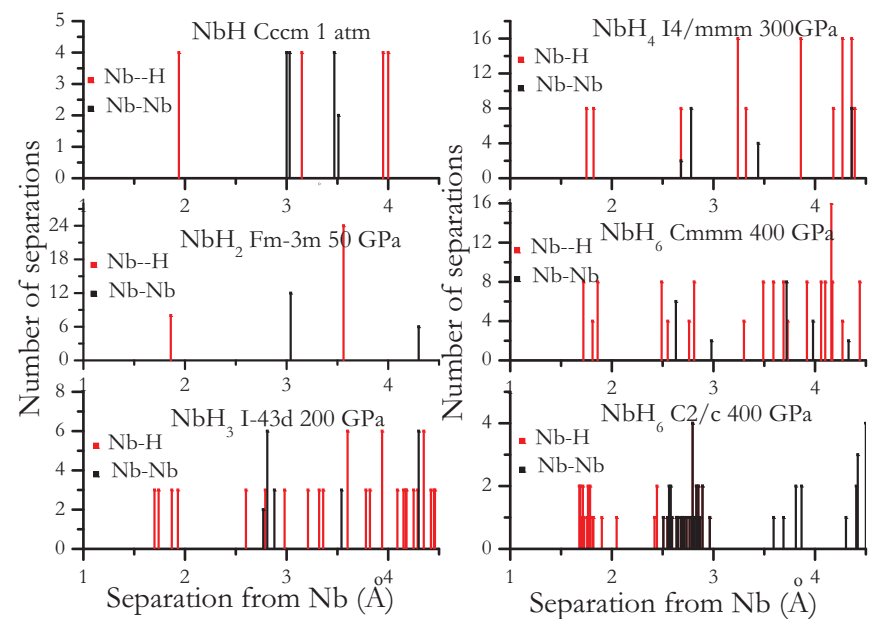

FIG. 17. (Color online) Histograms of $\mathrm{Nb}-\mathrm{H}$ (red) and $\mathrm{Nb}-\mathrm{Nb}$ (black) separations in $\mathrm{NbH}_{n}(n=1-4,6)$ each at a point in its enthalpically stable pressure region. Note that the vertical scale, showing the number of near neighbors at a given separation, is different for each phase. 
structures is thus not unreasonable in view of the fact that all hydrides in the extended structures exhibit double, triple, or even multibridging; such bridging M-H-M bonds in molecular hydrides are known to be longer than those for the terminal $\mathrm{M}-\mathrm{H}$ counterparts. For instance, as we mentioned earlier (and in Ref. 17), a terminal $\mathrm{Nb}-\mathrm{H}$ separation, $\mathrm{H}$ bonded to only a single $\mathrm{Nb}$, is $1.8 \AA$ [this is obtained from the neutron diffraction structure for $\left.\left.\mathrm{Cp}_{2} \mathrm{Nb}\left(\mathrm{SiHMe}_{2}\right)(\mathrm{H}) \mathrm{SiBrMe}_{2}\right)\right],{ }^{18}$ while a bridging $(\mathrm{Nb}-\mathrm{H}-\mathrm{Nb})$ separation is in the range of $1.8-2.12 \AA .{ }^{19,20}$

Of course, the higher $n$ is in $\mathrm{NbH}_{n}$, the larger will be in general the number of near $\mathrm{Nb}-\mathrm{H}$ separations. The histograms show a clear gap between a first group of $\mathrm{Nb}-\mathrm{H}$ separations of 1.70-1.94 $\AA$ (but for $\mathrm{NbH}_{6}$ there is a single $\mathrm{Nb}-\mathrm{H}$ separation at $2.1 \AA$ ), and the next longer group, of 2.60-3.56 $\AA$. This gap then allows us to define a plausibly unambiguous coordination number of hydrogens around a $\mathrm{Nb}$. With this criterion, we now obtain coordination numbers for $C c c m$ NbH, Fm-3m $\mathrm{NbH}_{2}, I-43 d \mathrm{NbH}_{3}, I 4 / \mathrm{mmm} \mathrm{NbH}$, and $C m m m \mathrm{NbH}_{6}$ of $4,8,12,16$, and 20, respectively (Fig. 18 shows the first coordination numbers). It is clear that in these ground-state structures the number of hydrogens around a niobium increases as $n$ in $\mathrm{NbH}_{n}$ increases, but it is intriguing to note that the number of hydrogens around each niobium in $\mathrm{NbH}_{n}$ ( $n=1-4)$ actually proceeds as $4 n$, and up to 20 in $\mathrm{NbH}_{6}$. We expect the coordination number to increase with pressure, but at this time, we do not have an explanation for the observed regularity.

Turning to the $\mathrm{Nb}-\mathrm{Nb}$ separations (the black lines in Fig. 17), the shortest ground-state $\mathrm{Nb}-\mathrm{Nb}$ separation, of $3.0 \AA$ in $\mathrm{NbH}$ at 1 atm, $3.04 \AA$ in $\mathrm{NbH}_{2}$ at $50 \mathrm{GPa}, 2.77 \AA$ in $\mathrm{NbH}_{3}$ at $200 \mathrm{GPa}, 2.68 \AA$ in $\mathrm{NbH}_{4}$ at $300 \mathrm{GPa}$ and $2.56 \AA$ in $\mathrm{NbH}_{6}$ at $400 \mathrm{GPa}$. These are longer than those in bulk Nb, namely, 2.88, $2.69,2.46,2.38$, and $2.32 \AA$, respectively, at corresponding pressures (see Fig. 19). It is obvious that the $\mathrm{Nb}$ lattice is expanded by incorporation of an increasing concentration of $\mathrm{H}$. This is consistent with the hydridic nature of the hydrogens in $\mathrm{NbH}_{n}$. Note that this occurs even though the average density increases. Within a given stoichiometry, the $\mathrm{Nb}-\mathrm{H}$ and $\mathrm{Nb}-\mathrm{Nb}$ separations do decrease with increasing pressure, when a large pressure range is specified.

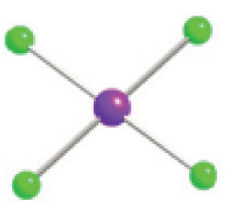

(a) $\mathrm{Cccm} \mathrm{NbH}$

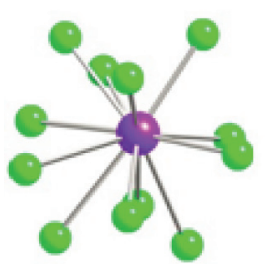

(c) $\mathrm{I}-43 \mathrm{~d} \mathrm{NbH}_{3}$

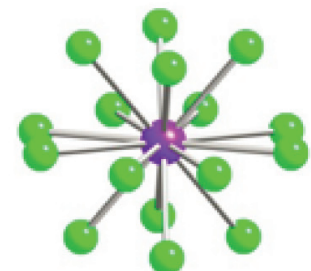

(d) $\mathrm{I} 4 / \mathrm{mmm} \mathrm{NbH}_{4}$

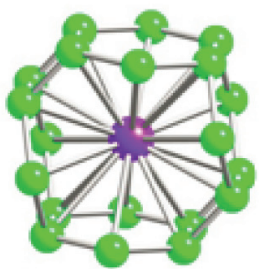

(e) $\mathrm{Cmmm} \mathrm{NbH}_{6}$
FIG. 18. (Color online) Hydrogen arrangements around each niobium in $\mathrm{NbH}_{n}(n=1-4,6)$.

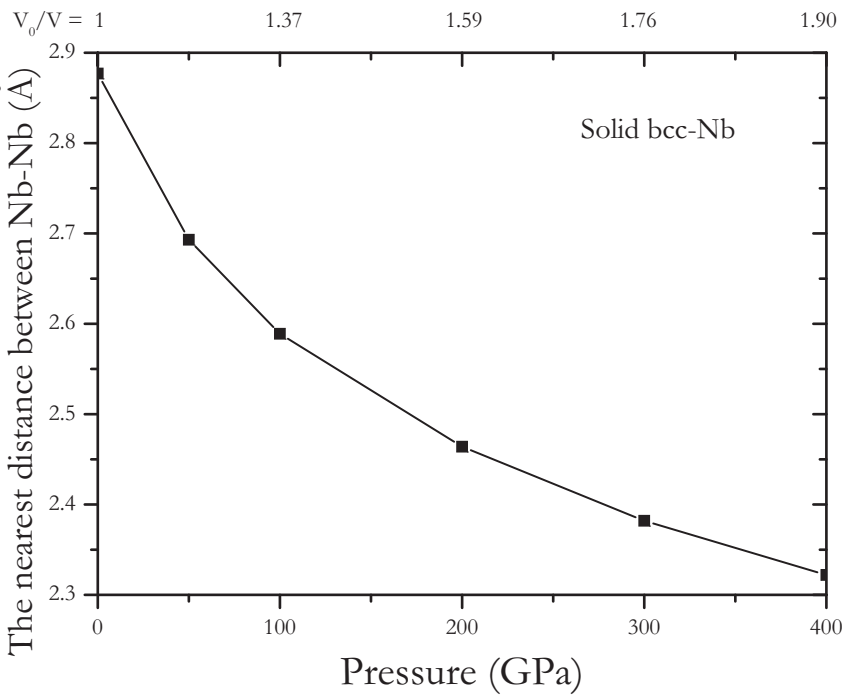

FIG. 19. The nearest $\mathrm{Nb}-\mathrm{Nb}$ separation at different pressures in pure solid and ground-state bcc-Nb. The relative compression $\left(V_{0} / V\right)$ is also presented for the corresponding pressures.

Comparing $\mathrm{NbH}_{n}(n=1-4,6)$ with our previously studied $\mathrm{WH}_{n}(n=1-6,8)$, we find many similarities: the near neighbor separations between $\mathrm{Nb}(\mathrm{W})$ and $\mathrm{H}$ atoms are all comparable to those computed or, indeed, found experimentally for the respective molecular hydrides. Moreover, the $\mathrm{Nb}-\mathrm{H}(\mathrm{W}-\mathrm{H})$ separations are not affected much by the hydrogen content of the hydride. The shortest $\mathrm{Nb}-\mathrm{Nb}$ separation of $2.8-3.05 \AA$ in niobium hydrides is thus very similar to the $\mathrm{W}-\mathrm{W}$ separations in tungsten hydrides ${ }^{9}$ of $2 .-3.0 \AA$ at the same pressure of $50 \mathrm{GPa}$. And both are a little longer than those found in the pure bcc-Nb, $2.7 \AA$ and bcc-W, $2.6 \AA$, respectively.

\section{N. The hydrogen sublattice}

The shortest $\mathrm{H}-\mathrm{H}$ separation in $\mathrm{NbH}_{\mathrm{n}}$ changes markedly with hydrogen content, again for static arrangements. To see
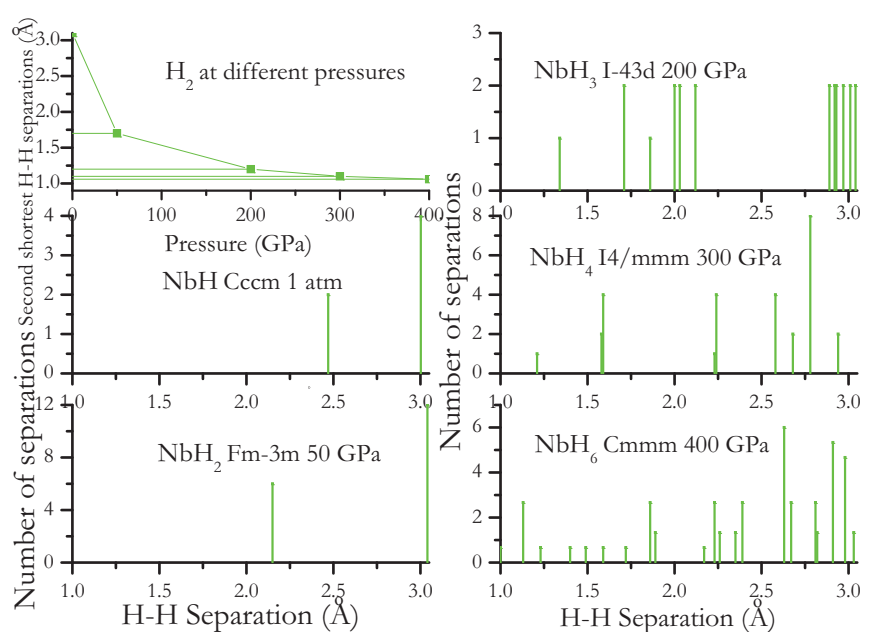

FIG. 20. (Color online) Histograms of $\mathrm{H}-\mathrm{H}$ separations in ground-state static $\mathrm{NbH}_{n}(n=1-4,6)$ each at a point in its enthalpically stable pressure region. Note that the vertical scale, the number of separations at specified distance, is different for each phase. 
this effect, and to separate it as much as possible from the effects of pressure, we compare the various stoichiometries, all at a single pressure $(50 \mathrm{GPa})$. Note that the higher hydrides are not stable at this pressure. As $n$ increases in $\mathrm{NbH}_{n}$, the shortest H-H separation decreases (see Fig. S16). ${ }^{17}$ Thus the shortest $\mathrm{H}-\mathrm{H}$ separation in $\mathrm{NbH}$ is $2.35 \AA$ at $50 \mathrm{GPa}$ in $\mathrm{NbH}_{2}$ $2.15 \AA$ in $\mathrm{NbH}_{3} 1.58 \AA$ in $\mathrm{NbH}_{4} 1.49 \AA$, and in $\mathrm{NbH}_{6}$ it is $1.07 \AA$. For comparison, the calculated second-shortest nearneighbor separation in $\mathrm{H}_{2}$ itself at the same pressure of $50 \mathrm{GPa}$ is $1.70 \AA .57$

What now transpires as pressure increases? As expected, the hydrogens in each structure approach each other (see Fig. 20); for instance, the shortest $\mathrm{H}-\mathrm{H}$ separation in $\mathrm{NbH}_{4}$ decreases down to $1.21 \AA$ at $300 \mathrm{GPa}$, and in $\mathrm{NbH}_{6}$ to even $1.00 \AA$ at $400 \mathrm{GPa}$. These are close to the second nearest separation in static pure solid hydrogen of $1.2 \AA$ at $200 \mathrm{GPa}$ and $1.06 \AA$ at $400 \mathrm{GPa}$, respectively. ${ }^{57}$ The shortest, bonding $\mathrm{H}-\mathrm{H}$ separations in $\mathrm{H}_{2}$ are $0.75-0.78 \AA$ at these pressures. We therefore again conclude that the electronic structures in these systems are leading to no paired hydrogens in the ground-state niobium hydrides studied, even at high pressures. However, there is definitely an extended $\mathrm{H}-\mathrm{H}$ bonding network in $\mathrm{NbH}_{4}$, and especially so in $\mathrm{NbH}_{6}$.

As the discussion of the attendant networks shows, the hydrogen arrangements are inherently three-dimensional and cannot be simply described. The situation thus differs markedly from $\mathrm{LiH}_{\mathrm{n}},{ }^{2}$ and also from $\mathrm{CaH}_{\mathrm{n}}{ }^{5}$ where for certain stoichiometries one clearly finds paired hydrogens. There is a definite connection to the $\mathrm{CaH}_{6}$ structure at $150 \mathrm{GPa},{ }^{5}$ which features an extended $\mathrm{H}$ network, albeit different from the ones we find here. We should also mention that it is certain that paired hydrogens will be found even at low pressures, if we were to examine a higher $n, n>6$ in $\mathrm{NbH}_{n}$ (as in the $\mathrm{W} / \mathrm{H}$ systems for $\mathrm{WH}_{8}{ }^{9}$ ). We have definitely found this in the preliminary calculations ${ }^{59}$ on $\mathrm{NbH}_{\mathrm{n}}$ mentioned earlier (where $n=12,24,48)$.

\section{CONCLUSIONS}

In the above, we have explored systematically the groundstate phases of selected niobium hydrides under pressure, both static and dynamic. The experimental structures for $\mathrm{Nb}_{4} \mathrm{H}_{3}$, $\mathrm{NbH}$, and $\mathrm{NbH}_{2}$ at 1 atm and relatively low temperature relate closely to those we have calculated. At higher pressures (e.g., $P \geqslant 50 \mathrm{GPa}$ ), $\mathrm{NbH}_{3}, \mathrm{NbH}_{4}$, and $\mathrm{NbH}_{6}$ become stable, each in their own range of pressures, and importantly, the initial pressure for stability in $\mathrm{NbH}_{4}$ and $\mathrm{NbH}_{6}$ will become progressively lower when we include the zero-point effects. It is very interesting to note that in the high-pressure structures of $\mathrm{NbH}_{3}$ and $\mathrm{NbH}_{4}$, the $\mathrm{Nb}$ atoms arrange themselves in a distorted bcc lattice and the $\mathrm{H}$ atoms occupy some of the available interstitial sites, while in the $\mathrm{NbH}_{6}$ structure, a very different structural principle is followed-the $\mathrm{H}$ atoms form a clathrate-type network with the $\mathrm{Nb}$ atoms in the channels.

Except for $\mathrm{NbH}_{5}$, all of the $\mathrm{Nb}$ hydrides studied are dynamically stable and also metallic, with reasonable values of the density of states at the Fermi level, which originates, as expected, mostly from the $\mathrm{Nb} 4 d$ orbitals. However, one also expects and in fact obtains contributions from the hydrogens as well in the higher of these hydrides, especially for $\mathrm{NbH}_{4}$ and $\mathrm{NbH}_{6}$. Interestingly, we find high-pressure $\mathrm{NbH}_{4}$ has the potential of being a good superconductor, with a preliminary estimate of $T_{c}$ around $40 \mathrm{~K}$ at $300 \mathrm{GPa}$, although the lower hydrides $\mathrm{NbH}$ and $\mathrm{NbH}_{2}$ might not become significant superconductors even at high pressure. This provides confirmation of our motivating points that adding a relatively small quantity of $\mathrm{Nb}$ may well induce insulating but dense hydrogen to enter the metallic state and become a potential higher temperature superconductor.

\section{ACKNOWLEDGMENTS}

We grateful to Andreas Hermann for discussions. G.G. and Y.M. acknowledge funding from the China 973 Program under Grant No. 2011CB808200, and National Natural Science Foundation of China under Grant Nos. 11274136, 11025418, and 91022029, the research fund of Key Laboratory of Surface Physics and Chemistry (Grant No. SPC201103). G.G., R.H., and N.W.A. acknowledge support by the National Science Foundation, through research grants CHE-0910623 and DMR-0907425, and EFree, an Energy Frontier Research Center funded by the U.S. Department of Energy (Award No. DESC0001057 at Cornell). Computational resources provided in part by Efree, TeraGrid, and XSEDE network (provided by the National Center for Supercomputer Applications through grant TG-DMR060055N). A.B. is grateful to the Department of Education, Universities and Research of the Basque Government, UPV/EHU (Grant No. IT-366-07) and the Spanish Ministry of Science and Innovation (Grant No. FIS2010-19609-C02-00) for financial support.

\footnotetext{
*Corresponding authors: rh34@ cornell.edu,

ashcroftnw@gmail.com, mym@jlu.ed.cn

${ }^{1}$ T. Skoskiewicz, Phys. Status Solidi A 11, K123 (1972); B. Stritzker and W. Buckel, Z. Physik 257, 1 (1972); K. H. Bennemann and J. W. Garland, ibid. 260, 367 (1973); B. M. Klein and R. E. Cohen, Phys. Rev. B 45, 12405 (1992).

${ }^{2}$ E. Zurek, R. Hoffmann, N. W. Ashcroft, A. R. Oganov, and A. O. Lyakhov, Proc. Natl. Acad. Sci. U.S.A. 106, 17640 (2009).

${ }^{3}$ P. Baettig and E. Zurek, Phys. Rev. Lett. 106, 237002 (2011).

${ }^{4}$ J. Hooper and E. Zurek, Chem.-Eur. J. 18, 5013 (2012).
}

${ }^{5}$ H. Wang, J. S. Tse, K. Tanaka, T. Iitaka, and Y. Ma, Proc. Natl. Acad. Sci. U.S.A. 109, 6463 (2012).

${ }^{6}$ P. Zaleski-Ejgierd, R. Hoffmann, and N. W. Ashcroft, Phys. Rev. Lett. 107, 037002 (2011).

${ }^{7}$ G. Y. Gao, A. R. Oganov, A. Bergara, M. Martinez-Canales, T. Cui, T. Iitaka, Y. Ma, and G. Zou, Phys. Rev. Lett. 101, 107002 (2008).

${ }^{8}$ G. Y. Gao, A. R. Oganov, P. Li, Z. Li, H. Wang, T. Cui, Y. Ma, A. Bergara, A. O. Lyakhov, and T. Iitaka, Proc. Natl. Acad. Sci. U.S.A. 107, 1317 (2010).

${ }^{9}$ P. Zaleski-Ejgierd, V. Labet, T. A. Strobel, R. Hoffmann, and N. W. Ashcroft, J. Phys.: Condens. Matter 24, 155701 (2012). 
${ }^{10}$ V. V. Struzhkin, Y. A. Timofeev, R. J. Hemley, and H. K. Mao, Phys. Rev. Lett. 79, 4262 (1997).

${ }^{11}$ L. R. Testardi, J. H. Wernick, and W. A. Royer, Solid State Commun. 15, 1 (1974).

${ }^{12}$ E. I. Isaev, S. I. Simak, and I. A. Abrikosov, J. App. Phys. 101, 123519 (2007).

${ }^{13}$ X.-J. Chen, V. V. Struzhkin, S. Kung, H. K. Mao, R. J. Hemley, and A. N. Christensen, Phys. Rev. B 70, 014501 (2004).

${ }^{14}$ A. E. Carlsson and N. W. Ashcroft, Phys. Rev. Lett. 50, 1305 (1983).

${ }^{15}$ X. F. Wang and L. Andrews, J. Phys. Chem. A 115, 14175 (2011).

${ }^{16}$ A. Otero, A. Antiiiolo, and A. Lara, in Comprehensive Organometallic Chemistry III, edited by R. H. Crabtree and D. M. P. Mingos (Oxford, 2007), p. 61.

${ }^{17}$ See Supplemental Material at http://link.aps.org/supplemental/ 10.1103/PhysRevB.88.184104 for the characteristic Nb-H separations in molecules, the crystal structure, dynamical stability, electronic and superconducting properties, histograms of $\mathrm{Nb}-\mathrm{H}$, $\mathrm{Nb}-\mathrm{Nb}$, and $\mathrm{H}-\mathrm{H}$, and the detailed structure information.

${ }^{18}$ K. Yu. Dorogov, M. Yousufuddin, N. N. Ho, A. V. Churakov, L. G. Kuzmina, A. J. Schultz, S. A. Mason, J. A. K. Howard, D. A. Lemenovskii, R. Bau, and G. I. Nikonov, Inorg. Chem. 46, 147 (2007).

${ }^{19}$ J. S. Figueroa, N. A. Piro, D. J. Mindiola, M. G. Fickes, and C. C. Cummins, Organometallics 29, 5215 (2010).

${ }^{20}$ F. Akagi, T. Matsuo, and H. Kawaguchi, Angew. Chem., Int. Ed. 46, 8778 (2007).

${ }^{21}$ K. M. Ho, H. J. Tao, and X. Y. Zhu, Phys. Rev. Lett. 53, 1586 (1984)

${ }^{22}$ A. Somenkov, A. V. Gurskaya, M. G. Zemlyanov, M. E. Kost, N. A. Chernoplekov, and A. A. Chertkov, Fiz. Tverd, Tela (Leningrad) 10, 1355 (1968) [Sov. Phys. Solid State 10, 1076 (1968)].

${ }^{23}$ T. Schober, M. A. Pick, and H. Wenzl, Phys. Status Solidi A 18, 175 (1973).

${ }^{24}$ D. J. Peterman, D. K. Misemer, J. H. Weaver, and D. T. Peterson, Phys. Rev. B 27, 799 (1983).

${ }^{25}$ J. J. Reilly and R. h. Wiseall, Inorg. Chem. 9, 1678 (1970).

${ }^{26}$ G. Brauer and H. Muller, Angew. Chem. 70, 53 (1958); G. Brauer and H. Muller, J. Inorg. Nucl. Chem. 17, 102 (1961).

${ }^{27}$ C. B. Satterthwaite and T. D. Peterson, J. Less-Common Met. 26, 361 (1972).

${ }^{28} \mathrm{We}$ have not estimated theoretically the entropy of the various structures.

${ }^{29}$ Y. Wang, J. Lv, L. Zhu, and Y. Ma, Phys. Rev. B 82, 094116 (2010).

${ }^{30}$ J. Lv, Y. Wang, L. Zhu, and Y. Ma, J. Chem. Phys. 137, 084104 (2012).

${ }^{31}$ Y. Wang, J. Lv, L. Zhu, and Y. Ma, Comput. Phys. Commun. 183, 2063 (2012).

${ }^{32}$ P. Li, G. Gao, Y. Wang, and Y. Ma, J. Phys. Chem. C 114, 21745 (2011)

${ }^{33}$ J. Lv, Y. Wang, L. Zhu, and Y. Ma, Phys. Rev. Lett. 106, 015503 (2011).

${ }^{34}$ L. Zhu, H. Wang, Y. Wang, J. Lv, Y. Ma, Q. Cui, Y. Ma, and G. Zou, Phys. Rev. Lett. 106, 145501 (2011).

${ }^{35}$ J. P. Perdew, K. Burke, and M. Ernzerhof, Phys. Rev. Lett. 77, 3865 (1996).
${ }^{36}$ G. Kresse and J. Furthmüler, Phys. Rev. B, 54, 11169 (1996).

${ }^{37}$ P. E. Blöchl, Phys. Rev. B 50, 17953 (1994).

${ }^{38}$ H. J. Monkhorst and J. D. Pack, Phys. Rev. B 13, 5188 (1976).

${ }^{39}$ K. Parlinski, Z. Q. Li, and Y. Kawazoe, Phys. Rev. Lett. 78, 4063 (1997).

${ }^{40}$ A. Togo, F. Oba, and I. Tanaka, Phys. Rev. B 78, 134106 (2008).

${ }^{41}$ P. Giannozzi, S. Baroni, N. Bonini, M. Calandra, M. Cococcioni, I. Dabo, A. DalCorso, S. D. Gironcoli, S. Fabris, G. Fratesi, R. Gebauer, U. Gerstmann, Christos Gougoussis, A. Kokalj, M. Lazzeri, L. Martin-samos, N. Marzari, F. Mauri, R. Mazzarello, S. Paolini, A. Pasquarello, L. Paulatto, C. Sbraccia, A. Smogunov, P. Umari, and R. M. Wentzcovitch, J. Phys. Condens. Matter 21, 395502 (2009).

${ }^{42}$ P. B. Allen and R. Silberglitt, Phys. Rev. B 9, 4733 (1974).

${ }^{43}$ P. B. Allen, Phys. Rev. B 6, 2577 (1972).

${ }^{44}$ J. R. Schrieffer, The Theory of Superconductivity (Benjamin, New York, 1964).

${ }^{45}$ P. B. Allen and R. C. Dynes, Phys. Rev. B 12, 905 (1975).

${ }^{46}$ Experimental photoemission spectra are available for $\mathrm{NbH}_{x}(21)$. We have resisted using these as a way to distinguish the various computed structures because the photoemission is likely to be a surface and not a bulk phenomenon.

${ }^{47}$ G. Y. Gao, H. Wang, L. Zhu, and Y. M. Ma, J. Phys. Chem. C 116, 1995 (2012).

${ }^{48}$ C. J. Pickard and R. J. Needs, Nat. Phys. 3, 473 (2007).

${ }^{49}$ A. Fukizawa and Y. Fukai, J. Phys. Soc. Jpn. 52, 2102 (1983).

${ }^{50}$ H. Taguchi, Y. Fukai, T. Atou, K. Fukuoka, and Y. Syono, Phys. Rev. B 49, 3025 (1993).

${ }^{51}$ A. F. Andresen, A. J. Maeland, and D. Slotfeldt-Ellingsen, J. Solid State Chem. 20, 93 (1977).

${ }^{52}$ Y. Li, B. Li, T. Cui, Y. Li, L. Zhang, Y. Ma, and G. Zou, J. Phys.: Condens. Matter 20, 045211 (2008).

${ }^{53}$ K. Kinoshita, M. Nishimura, Y. Akahama, and H. Kawamura, Solid State Commun. 141, 69 (2007).

${ }^{54}$ Y. S. Yao and D. D. Klug, Phys. Rev. B 81, 140104(R) (2010).

${ }^{55}$ I. Goncharenko, M. I. Eremets, M. Hanfland, J. S. Tse, M. Amboage, Y. Yao, and I. A. Trojan, Phys. Rev. Lett. 100, 045504 (2008).

${ }^{56}$ X. L. Jin, X. Meng, Z. He, Y. M. Ma, B. B. Liu, T. Cui, G. T. Zou, and H. K. Mao, Proc. Natl. Acad. Sci. U.S.A. 107, 9969 (2010).

${ }^{57}$ V. Labet, P. Gonzalez-Morelos, R. Hoffmann, and N. W. Ashcroft, J. Chem. Phys. 136, 074501 (2012).

${ }^{58}$ K. Nagao, S. A. Bonev, A. Bergara, and N. W. Ashcroft, Phys. Rev. Lett. 90, 035501 (2003).

${ }^{59}$ G. Y. Gao, N. Ashcroft, and R. Hoffmann (unpublished).

${ }^{60}$ Y. S. Yao, D. D. Klug, J. Sun, and R. Martonak, Phys. Rev. Lett. 103, 055503 (2009).

${ }^{61}$ K. Iyakutti and C. N. Louis, Phys. Rev. B 70, 132504 (2004).

${ }^{62}$ K. Shimizu, H. Ishikawa, D. Takao, T. Yagi, and K. Amaya, Nature 419, 597 (2002).

${ }^{63}$ J. S. Tse, D. D. Klug, S. Patchkovskii, Y. M. Ma, and J. K. Dewhurst, J. Phys. Chem. B 110, 3721 (2006).

${ }^{64}$ G. Y. Gao, H. Wang, A. Bergara, Y. W. Li, G. T. Liu, and Y. M. Ma, Phys. Rev. B 84, 064118 (2011). 